\title{
THE
}

\section{Estimation of Directional Surface Wave Spectra from a Towed Research Catamaran}

Kurt A. Hanson

University of Rhode Island

Tetsu Hara

University of Rhode Island, thara@uri.edu

Erik J. Bock

Andrew B. Karachintsev

Follow this and additional works at: https://digitalcommons.uri.edu/gsofacpubs

\section{Citation/Publisher Attribution}

Hanson, K.A., T. Hara, E.J. Bock, and A.B. Karachintsev, 1997: Estimation of Directional Surface Wave Spectra from a Towed Research Catamaran*. J. Atmos. Oceanic Technol., 14, 1467-1482. doi: 10.1175/ 1520-0426(1997)0142.0.CO;2

Available at: https://doi.org/10.1175/1520-0426(1997)014<1467:EODSWS>2.0.C0;2

This Article is brought to you for free and open access by the Graduate School of Oceanography at DigitalCommons@URI. It has been accepted for inclusion in Graduate School of Oceanography Faculty Publications by an authorized administrator of DigitalCommons@URI. For more information, please contact digitalcommons-group@uri.edu. 


\title{
Estimation of Directional Surface Wave Spectra from a Towed Research Catamaran*
}

\author{
Kurt A. Hanson ${ }^{+}$AND Tetsu Hara \\ Graduate School of Oceanography, University of Rhode Island, Narrangansett, Rhode Island \\ ERIK J. BOCK \\ Woods Hole Oceanographic Institution, Woods Hole, Massachusetts \\ ANDREY B. KARACHINTSEV \\ Graduate School of Oceanography, University of Rhode Island, Narrangansett, Rhode Island
}

(Manuscript received 16 August 1996, in final form 3 April 1997)

\begin{abstract}
During the High-Resolution Remote Sensing Main Experiment (1993), wave height was estimated from a moving catamaran using pitch-rate and roll-rate sensors, a three-axis accelerometer, and a capacitive wave wire. The wave spectrum in the frequency band ranging roughly from 0.08 to $0.3 \mathrm{~Hz}$ was verified by independent buoy measurements. To estimate the directional frequency spectrum from a wave-wire array, the Data-Adaptive Spectral Estimator is extended to include the Doppler shifting effects of a moving platform. The method is applied to data obtained from a fixed platform during the Ris $\varnothing$ Air-Sea Experiment (1994) and to data obtained from a moving platform during the Coastal Ocean Processes Experiment (1995). Both results show that the propagation direction of the peak wind waves compares well with the measured wind direction. When swells and local wind waves are not aligned, the method can resolve the difference of propagation directions. Using the fixed platform data a numerical test is conducted that shows that the method is able to distinguish two wave systems propagating at the same frequency but in two different directions.
\end{abstract}

\section{Introduction}

In the past several years research catamarans were used in various in situ measurements of small-scale airsea interaction processes, including wind waves, atmospheric turbulence, subsurface turbulence, and surface films (Carlson et al. 1988; Bock and Frew 1993; Hara et al. 1994; Bock et al. 1995). Such platforms have the advantage of having very low profiles, hence they generate little disturbance on processes of interest, in contrast to traditional shipboard measurements. Furthermore, they do not contaminate surrounding water surfaces as long as no oil-based engines are installed. This is an essential requirement for studies on smallscale processes that are easily affected by the presence of surfactants. Examples of sensors mounted on research

\footnotetext{
* WHOI Contribution Number 9311.

+ Current affiliation: Center for Coastal Geology, U.S. Geological Survey, St. Petersburg, Florida.
}

Corresponding author address: Dr. Tetsu Hara, Graduate School of Oceanography, University of Rhode Island, Narragansett, RI 02882 .

E-mail: tetsu@ripples.gso.uri.edu catamarans include an acoustic anemometer, a (scanning) laser slope gauge to measure short wind waves, a surface skimmer to measure surface enrichment, an acoustic current meter, and a hot-film anemometer to measure subsurface currents and/or turbulence.

In addition to measuring various processes independently, some of the recent efforts focus on the interaction among different physical-chemical processes at the airsea interface. Examples include the effect of gravity waves on gravity-capillary waves, and the coupling between surface gravity waves and near-surface wind turbulence. These studies require simultaneous and collocated measurements of surface gravity waves with other measurements of small-scale processes. Although directional wind-wave spectra alone have been measured successfully from a buoy (Longuet-Higgins et al. 1963; Kats and Spevak 1980) or from a fixed platform (Donnelan et al. 1985), it is of great interest to achieve similar measurements from a research catamaran in order to integrate the results with other measurements.

Recently, Drennan et al. (1994) have demonstrated that it is possible to estimate directional wind-wave spectra from a swath ship. First, they apply the motion correction to the signals from a wave-wire array mounted at the bow of the ship. They then apply the maximum 
likelihood method to estimate the directional spreading of wave energy. Motivated by their study, we have attempted to obtain directional wave spectra by combining signals from pitch-rate and roll-rate sensors, a three-axis accelerometer, and an array of capacitive wave wires, all mounted on a research catamaran. Although the basic principle of our data analysis scheme is similar to that in Drennan et al. (1994), a more careful motion correction scheme is needed because the motion of the catamaran is substantially more affected by surface waves. In addition, we have extended the Data-Adaptive Spectral Estimator (DASE) of Davis and Regier (1977) to estimate the directional frequency spectrum of wind waves including the presence of Doppler shifting. This method explicitly makes use of the dispersion relation of surface gravity waves and reduces the degrees of freedom compared with the maximum likelihood method used in Drennan et al. (1994).

In section 2, we describe the motion correction to the wave-wire signals. The scheme is applied to signals obtained during High-Resolution Remote Sensing Main Experiment (Hi-Res 2) of 1993 in section 3. The results are confirmed by comparison with independent buoy measurements of wind-wave spectra.

In section 4, we extend DASE to estimate the directional frequency spectrum of surface gravity waves including the presence of Doppler shifting. The scheme is first applied to data obtained during the Ris $\varnothing$ Air-Sea Experiments (RASEX) of 1994 in section 5. In addition, numerical experiments are conducted to investigate how the method can distinguish secondary waves that propagate in a different direction from dominant waves. Finally, in section 6 , the method of motion correction and DASE are combined to estimate directional wave spectra from a moving research catamaran, using data obtained during the Coastal Ocean Processes Experiments (CoOP) of 1995.

\section{Wave-height measurement from a moving platform}

In this section we describe a method of obtaining a true wave height signal by combining signals from a motion detection package and a wave wire. Both instruments are mounted on a research catamaran that is towed alongside a research vessel.

\section{a. Coordinate systems}

Two reference frames are used in section 2. Since the instruments are mounted on a moving catamaran, we define a frame fixed to the platform as the "rotating frame," with the positive $x_{r}$ axis in the forward direction and the positive $y_{r}$ axis pointing to port. The $z_{r}$ axis is defined to be along the unit vector $\mathbf{k}_{r}=\mathbf{i}_{r} \times \mathbf{j}_{r}$, where $\mathbf{i}_{r}$ and $\mathbf{j}_{r}$ are the unit vectors along the $x_{r}$ and $y_{r}$ axes, respectively; that is, the positive $z_{r}$ axis is upward. The other coordinate system used for analyses is the "non-

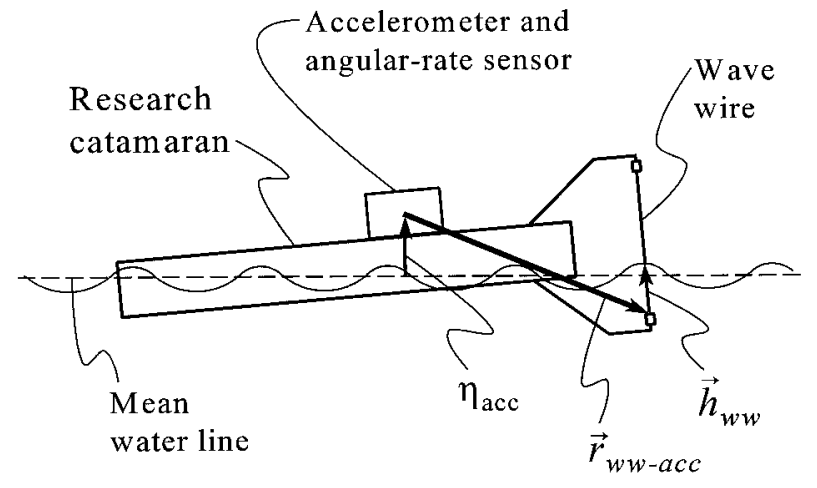

FIG. 1. Schematic showing wave height estimation from the research catamaran.

rotating frame" with axes $x_{c}, y_{c}$, and $z_{c}$ defined by the long time mean (in terms of wave-induced motions) of the rotating-frame axes. The mean water level is at $z_{c}$ $=0$. The $x_{c}, y_{c}$, and $z_{c}$ axes are parallel to the unit vectors $\mathbf{i}_{c}, \mathbf{j}_{c}$, and $\mathbf{k}_{c}$, respectively. Time series for our analyses are selected only if they do not contain large changes in the speed and direction of the platform and in the mean current velocity. The nonrotating frame is then approximately equivalent to a fixed reference moving with a constant velocity relative to the mean current field. This criterion is verified by analyzing the velocity measured by the current meter. For the time series analyzed, the standard deviations of the $x_{r}$ and $y_{r}$ components of the current-meter velocity are typically 0.1 $\mathrm{m} \mathrm{s}^{-1}$, or about $15 \%$ of their means. We may also characterize the nonrotating frame by analyzing the roll, pitch, and yaw motions of the catamaran about the $x_{c}$, $y_{c}$, and $z_{c}$ axes, respectively. The standard deviations of these angles are about $4^{\circ}$ for pitch and roll and $9^{\circ}$ for yaw.

\section{b. Characterization of wave height}

The wave wires measure wave height relative to the moving catamaran. The catamaran motion is sensed by the accelerometers and angular-rate sensors. These wave wire and motion package data are combined to yield wave height relative to the mean sea surface $\eta$ according to the equation

$$
\eta=\eta_{\mathrm{ww}}+\eta_{\mathrm{ww}-\mathrm{acc}}+\eta_{\mathrm{acc}},
$$

where $\eta_{\mathrm{ww}}$ (vertical component of $\mathbf{h}_{\mathrm{ww}}$ ) is the sea surface height relative to the base of the wave wire, $\eta_{\mathrm{ww} \text {-acc }}$ (vertical component of $\mathbf{r}_{\mathrm{ww}-\mathrm{acc}}$ ) is the height of the base of the wave wire relative to the accelerometers (which is negative), and $\eta_{\text {acc }}$ is the height of the accelerometers relative to the mean sea surface (see Fig. 1). These heights are expressed in the nonrotating reference frame $\left(x_{c}, y_{c}, z_{c}\right)$ defined in section 2a.

Wave height is calculated using a multistep procedure outlined in Fig. 2. The $\eta_{\mathrm{ww}}$ term in (1) is given directly by the wave wire. The heights $\eta_{\mathrm{ww}-\mathrm{acc}}$ and $\eta_{\mathrm{acc}}$ depend 


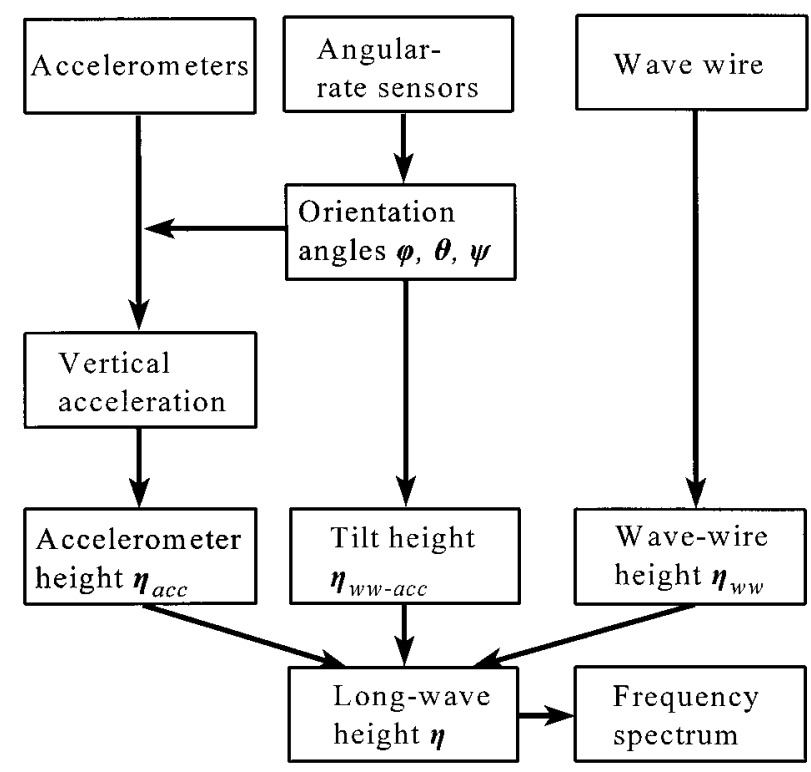

FIG. 2. Flowchart of the wave-height estimation procedure.

on the pitch and roll angles $\theta$ and $\phi$. These angles are computed by integrating the pitch and roll rates measured by the angular-rate sensors. The acceleration vector measured by the accelerometers is then converted from the rotating frame to the nonrotating frame using $\theta$ and $\phi$. The vertical acceleration $\ddot{z}_{c}$ is integrated twice, resulting in the height $\eta_{\text {acc }}$ shown in Fig. 1. The height $\eta_{\mathrm{ww}-\mathrm{acc}}$ is the vertical component of the position vector $\mathbf{r}_{\mathrm{ww}-\mathrm{acc}}$ from the accelerometers to the base of the wave wires (see Fig. 1). It is found by transforming $\mathbf{r}_{\mathrm{ww}-\mathrm{acc}}$ from the rotating to the nonrotating frame using $\theta$ and $\phi$.

\section{c. Transformation matrix}

The accelerometer signals and the position vector $\mathbf{r}_{\mathrm{ww}-\mathrm{acc}}$ are measured in the rotating frame $\left(x_{r}, y_{r}, z_{r}\right)$. These must be expressed in the nonrotating frame $\left(x_{c}\right.$, $y_{c}, z_{c}$ ) to compute the heights $\eta_{\mathrm{acc}}$ and $\eta_{\mathrm{ww}-\mathrm{acc}}$. A transformation matrix is used for this conversion. Consider a reference frame $(x, y, z)$ that undergoes three separate rotations about each of its own axes. In general, the final orientation of the frame depends upon the order in which the axis rotations are made. For the case of rotation through angles $\psi, \theta$, and $\phi$, in that order, the transformation matrix

$$
\begin{aligned}
\mathbf{T}_{\psi \theta \phi}= & \left(\begin{array}{ccc}
\cos \psi & -\sin \psi & 0 \\
\sin \psi & \cos \psi & 0 \\
0 & 0 & 1
\end{array}\right)\left(\begin{array}{ccc}
\cos \theta & 0 & \sin \theta \\
0 & 1 & 0 \\
-\sin \theta & 0 & \cos \theta
\end{array}\right) \\
& \times\left(\begin{array}{ccc}
1 & 0 & 0 \\
0 & \cos \phi & -\sin \phi \\
0 & \sin \phi & \cos \phi
\end{array}\right)
\end{aligned}
$$

relates the final orientation of the axes to the original orientation (Macomber and Fernandez 1962). Note that the angles $\psi, \theta$, and $\phi$ refer to rotation angles about the $z, y$, and $x$ axes of the frame undergoing rotation, where positive angles are described by the right-hand rule. The transformation matrix for any alternate order of axis rotations is found through rearrangement of the matrices on the right-hand side of (2). However, when rotation angles are small, the transformation matrix becomes approximately independent of the order of rotation. In this case, (2) becomes

$$
\mathbf{T}_{\mathrm{sm}}=\left(\begin{array}{ccc}
1 & -\psi & \theta \\
\psi & 1 & -\phi \\
-\theta & \phi & 1
\end{array}\right),
$$

where the angles $\phi, \theta$, and $\psi$ are now to be expressed in radians. Equation (3) is used to convert from the rotating frame $\left(x_{r}, y_{r}, z_{r}\right)$ to the nonrotating frame $\left(x_{c}\right.$, $\left.y_{c}, z_{c}\right)$. The rotating frame is equivalent to the nonrotating frame when the latter is rotated through angles $\phi, \theta$, and $\psi$ about its axes.

\section{d. Orientation angles}

The angular-rate signals are first converted from voltage to angular rates in radians per second using a linear calibration. The angular-rate sensor signals contain "drift" error appearing as noise at low frequencies. Thus, we subtract the best-fit linear trend from each 10-min time series. The trend was on average less than $0.5 \%$ of the standard deviation of the angles with trend removed. The pitch, roll, and yaw angles $\theta, \phi$, and $\psi$ are then computed from the rate signals using a numerical integration scheme with third-order accuracy. The form of this scheme for the general, discrete variable $a_{i}$ is given by

$$
a_{i}=a_{i-1}+\frac{1}{f_{\text {samp }}}\left(\frac{1}{4} \dot{a}_{i}+\dot{a}_{i-1}+\frac{1}{4} \dot{a}_{i-2}\right)+O\left(f_{\text {samp }}^{-3}\right),
$$

where $i$ is the index of the discrete $a, \dot{a}$ represents the time derivative of $a$, and $f_{\text {samp }}$ is the sampling frequency. This is the Taylor series approximation with third-order accuracy, where the term $\ddot{a}$ is replaced with the central difference approximation (see, e.g., Hornbeck 1975).

\section{e. Accelerometer height}

The height of the accelerometers relative to the mean sea surface $\eta_{\text {acc }}$ is estimated using the pitch and roll angles. This calculation requires several steps. First, a linear calibration is applied to the voltage outputs of the accelerometers, yielding acceleration signals $\mathbf{x}_{\mathrm{obs}}$ in meters per second squared. Next, a linear trend is removed from each 10-min signal. The average magnitude of this trend is $1 \%$ or less of the standard deviation of the signal. The accelerometer signals are scaled for consistency with the magnitude of the gravity vector. The scale 
factor is the ratio of $9.81 \mathrm{~m} \mathrm{~s}^{-2}$ to the 10 -min mean acceleration magnitude and averages $0.965 \pm 0.004$.

The vertical acceleration in the nonrotating frame $\ddot{z}_{\text {acc }}$ is computed using the accelerometer signals $\ddot{\mathbf{x}}_{\text {obs }}$ and the small-angle transformation matrix, according to

$$
\ddot{z}_{\mathrm{acc}}=\left(\mathbf{T}_{\mathrm{sm}} \ddot{\mathbf{x}}_{\mathrm{obs}}\right) \cdot \mathbf{k}_{c} \text {. }
$$

Substituting for $\mathbf{T}_{\mathrm{sm}}$ using (3) yields

$$
\ddot{z}_{\mathrm{acc}}=-\theta \ddot{x}_{\mathrm{obs}}+\phi \ddot{y}_{\mathrm{obs}}+\ddot{z}_{\mathrm{obs}},
$$

where $\ddot{x}_{\mathrm{obs}}, \ddot{y}_{\mathrm{obs}}$, and $\ddot{z}_{\mathrm{obs}}$ are the $x_{r}, y_{r}$, and $z_{r}$ components of $\ddot{\mathbf{x}}_{\text {obs }}$. The $\ddot{z}_{\text {acc }}$ signal is then integrated twice in the time domain using the Taylor series approximation of (4), yielding the accelerometer height $\eta_{\text {acc }}$ of (1). Prior to each integration, the linear trend over $10 \mathrm{~min}$ is removed.

\section{f. Tilt height}

The pitch and roll angles are also used in the estimation of the tilt height $\eta_{\mathrm{ww}-\mathrm{acc}}$, which is the height of the base of the wave wire relative to the accelerometer. This height is related to $\mathbf{r}_{\mathrm{ww}-\mathrm{acc}}$, which is the vector from the accelerometer to the base of the wave wire, through the transformation matrix $\mathbf{T}_{\mathrm{sm}}$;

$$
\eta_{\mathrm{ww}-\mathrm{acc}}=\left(\mathbf{T}_{\mathrm{sm}} \mathbf{r}_{\mathrm{ww}-\mathrm{acc}}\right) \cdot \mathbf{k}_{c}
$$

where $\mathbf{r}_{\mathrm{ww}-\mathrm{acc}}$ is a fixed vector in the rotating frame. Equation (3) is used to simplify (7) to

$$
\eta_{\mathrm{ww}-\mathrm{acc}}=-\theta x_{\mathrm{ww}-\mathrm{acc}}+\phi y_{\mathrm{ww}-\mathrm{acc}}+z_{\mathrm{ww}-\mathrm{acc}},
$$

where $x_{\mathrm{ww}-\mathrm{acc}}, y_{\mathrm{ww}-\mathrm{acc}}$, and $z_{\mathrm{ww}-\mathrm{acc}}$ are the $x_{r}, y_{r}$, and $z_{r}$ components, respectively, of the column vector $\mathbf{r}_{\mathrm{ww}-\mathrm{acc}}$.

\section{g. Wave-wire height}

The last unknown in the computation of sea surface height given by (1) is the wave-wire height $\eta_{\mathrm{ww}}$. Its calculation is analogous to the tilt-height estimate of (7) and is given by

$$
\eta_{\mathrm{ww}}=\left(\mathbf{T}_{\mathrm{sm}} \mathbf{h}_{\mathrm{ww}}\right) \cdot \mathbf{k}_{c} .
$$

Here, $\mathbf{h}_{\mathrm{ww}}$ is the vector from the base of the wave wire to the intersection of the wire with the sea surface. The multiplication is trivial since the only nonzero component of $\mathbf{h}_{\mathrm{ww}}$ is the $z_{c}$ component, which is the wavewire signal $z_{\mathrm{ww}}$. By substituting for $\mathbf{T}_{\mathrm{sm}}$ using (3), (9) becomes

$$
\eta_{\mathrm{ww}}=z_{\mathrm{ww}}
$$

\section{Application of motion correction scheme}

\section{a. Overview of Hi-Res 2 experiment}

The main experiment of the High-Resolution Remote Sensing Program (Hi-Res 2) occurred in June 1993 and was sponsored by the Office of Naval Research/Naval

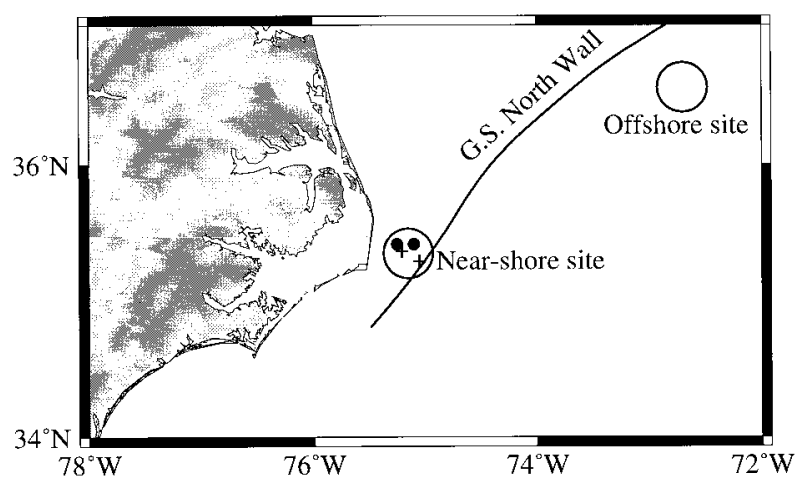

FIG. 3. The location of the High-Res 2 experiment, near the barrier islands of North Carolina. Ship locations are shown as crosses. The east and west discus buoys are indicated by filled circles. The average location of the Gulf Stream north wall is shown.

Research Laboratory. The principal objective of the program was to investigate the accuracy of radar measurements of submesoscale processes at the ocean surface. The research catamaran was deployed as a means of obtaining in situ measurements of gravity-capillary waves (using a scanning laser slope gauge), surface gravity waves (using a capacitive wave wire, and a motion detection package), near-surface atmospheric turbulence (using a three-axis acoustic anemometer mounted at 5-m mean altitude), and subsurface currents (using a three-axis acoustic Doppler current meter at 1-m mean depth). The catamaran was towed to the side of the R/V Iselin at a distance of about $20 \mathrm{~m}$ in order to minimize effects due to the wind blockage and ship wake of the vessel. Data from the wave wire and the motion detection package were recorded digitally at a sampling rate of $20 \mathrm{~Hz}$.

The location of the experiment was near the coast of Cape Hatteras, North Carolina. During the experiment the position of the ship was recorded with an onboard GPS sensor. The two main experimental sites are shown in Fig. 3 by large circles. One site near $36.6^{\circ} \mathrm{N}, 72.8^{\circ} \mathrm{W}$ was called "offshore site"; the other site was called "nearshore site." Both sites were located in the vicinity of the Gulf Stream north wall so that frontal features could be investigated. In the following section we show results obtained from only the nearshore site. Also shown in Fig. 3 are the locations of two discus buoys (filled circles). These buoys were deployed by a group led by Dr. H. Graber of the Rosenstiel School of Marine and Atmospheric Sciences at the University of Miami.

\section{b. Results of wave height frequency spectra}

Two examples of wave height frequency spectra, calculated using the method of the appendix, are shown in Figs. 4 and 5. These spectra were computed using 161-s time blocks and total time series lengths of 121 and 90 min, respectively. The corresponding ship tracks are indicated in Fig. 3. In Figs. 4 and 5 the $95 \%$ confidence 


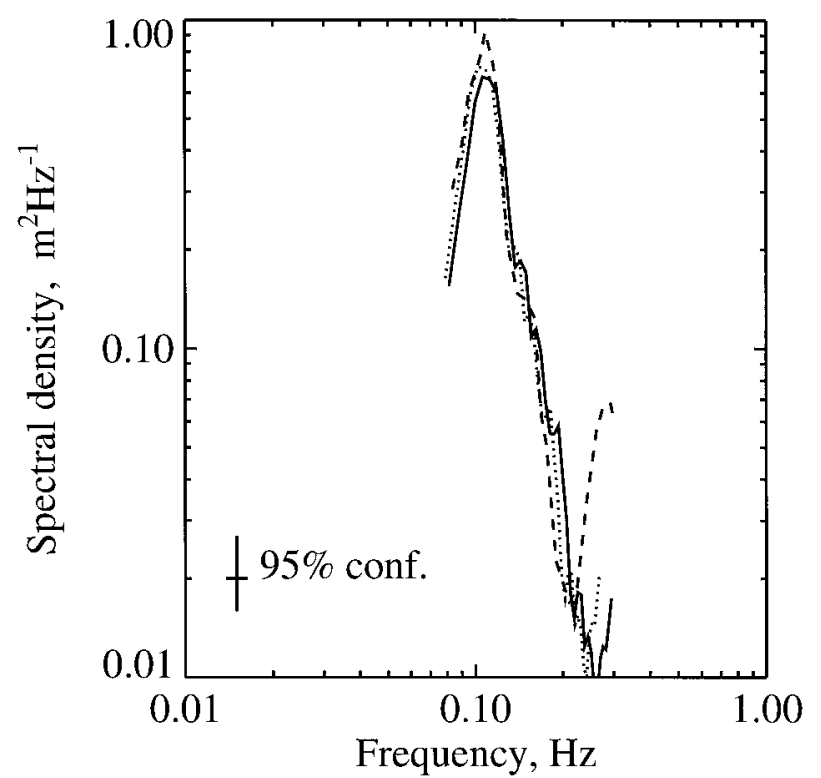

FIG. 4. High-Res 2 wave-height frequency spectrum for Julian day 171 from 1510 to 1711 UTC $(P=60$ time blocks $)$. Here, $u_{*}=0.21$ $\mathrm{m} \mathrm{s}^{-1}$ and $U_{10}=6.4 \mathrm{~m} \mathrm{~s}^{-1}$. Solid: without Doppler shifting correction. Dotted: with unidirectional Doppler shifting correction. Dashed: west discus buoy spectrum at 1600 UTC.

limits are shown as vertical bars. These limits describe the statistical stability of the spectral estimates and are given approximately by

$$
\frac{1}{1+2 / \sqrt{P}} \hat{C}_{\eta \eta}(f) \leq C_{\eta \eta}(f) \leq \frac{1}{1-2 / \sqrt{P}} \hat{C}_{\eta \eta}(f),
$$

where $C_{\eta \eta}(f)$ and $\hat{C}_{\eta \eta}(f)$ are the true and estimated frequency spectra, and $P$ is the number of time blocks used in the spectral calculations. A more exact form of (11) is found by replacing $P$ with the number of degrees of freedom. Because overlapping time blocks are used in the spectral calculations, the exact number of degrees of freedom is unknown but is within the range $P / 2$ to $P$.

Our frequency spectra may be compared to frequency spectra derived from the two discus buoys. The spectrum from the buoy closest to the position of the catamaran is shown as a dashed line in Figs. 4 and 5. The frequencies of the swell peaks (near $0.1 \mathrm{~Hz}$ ) and the swell-peak spectral densities agree reasonably well in both cases. In Fig. 5 local wind-wave peaks near 0.2 $\mathrm{Hz}$ are indicated in both the catamaran and buoy spectra. However, the wind-wave peak spectral levels are larger in the buoy spectra than in the catamaran spectra by factors of about 2. This discrepancy may be partly attributable to the differences in the location and in the time of the two measurements.

The catamaran spectra are affected by Doppler shifting. A simple means of accounting for Doppler shifting is to assume that the wave field is completely unidirectional and moves toward the catamaran along its forward

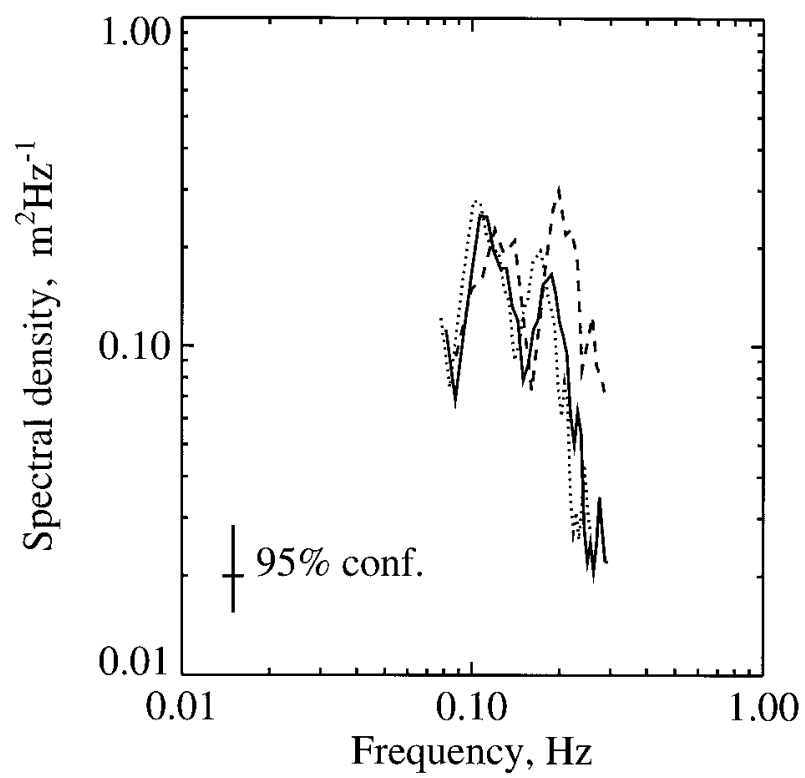

FIG. 5. High-Res 2 wave-height frequency spectrum for Julian day 173 from 1918 to 2048 UTC ( $P=45$ time blocks). Here, $u_{*}=0.24$ $\mathrm{m} \mathrm{s}^{-1}$ and $U_{10}=8.0 \mathrm{~m} \mathrm{~s}^{-1}$. Solid: without Doppler shifting correction. Dotted: with unidirectional Doppler shifting correction. Dashed: east discus buoy spectrum at 2000 UTC.

axis. This assumption represents the largest possible Doppler shift, from a given intrinsic frequency $\tilde{f}$ to the observed frequency $f$. The catamaran velocity relative to the mean current in the forward direction uniquely determines the Doppler shifting of the waves. This velocity is calculated using a long-term average of the current meter velocity along the $x_{c}$ axis. The ordinate of $\hat{C}_{\eta \eta}$ is converted from the observed frequency $f$ to the intrinsic frequency $\tilde{f}$ using the Doppler-shifted dispersion relation

$$
\tilde{f}=\frac{g}{4 \pi U_{\mathrm{obs}}}\left(\sqrt{1+8 \pi U_{\mathrm{obs}} f / g}-1\right),
$$

where $g$ is the gravitational acceleration and $U_{\text {obs }}$ is the speed of the catamaran relative to the mean current. Furthermore, the $\hat{C}_{\eta \eta}(\tilde{f})$ values must be scaled so that the total wave energy remains the same after the transformation of (12). This scale factor is $\delta f / \delta \tilde{f}$ where $\delta f$ is the frequency resolution and $\delta \tilde{f}$ is the intrinsic frequency resolution, defined by

$$
\begin{aligned}
\delta \tilde{f} \equiv \frac{g}{4 \pi U_{\text {obs }}}[ & {\left[\sqrt{1+8 \pi U_{\text {obs }}(f+\delta f / 2) / g}\right.} \\
& \left.-\sqrt{1+8 \pi U_{\text {obs }}(f-\delta f / 2) / g}\right] .
\end{aligned}
$$

The results of Figs. 4 and 5 (dotted lines) show that Doppler shifting is quite small for the frequency bandwidth described.

\section{c. Accuracy of wave-height results}

We have found that the region consisting of frequencies less than about $0.08 \mathrm{~Hz}$ is contaminated by noise 
from the $z_{r}$ accelerometer. Near $0.5 \mathrm{~Hz}$, there is error due to the resonant motion of the catamaran [see Hanson (1996) for detailed analyses]. We therefore focus on the region of the frequency spectrum from roughly 0.08 to $0.3 \mathrm{~Hz}$, shown in Figs. 4 and 5.

The voltage outputs of the accelerometers and angular-rate sensors are linearly proportional to the quantities that they measure. They are accurate to within 3\%, provided that the physical quantities do not exceed the maximum measurement ranges of the instruments. Time series for which this occurred were excluded from the analyses. The wave-wire outputs are also linear and accurate to less than $1 \%$.

The wave height result is derived by assuming that the catamaran orientation angles are small, so that the transformation matrix of (3) may be used. The smallangle requirement is met for yaw angle by selecting time series for analysis without large catamaran turns. The standard deviation of compass angle was less than $10^{\circ}$ for the spectra shown. The yaw angles had standard deviations similar to those of the compass angles. The pitch and roll angles were inspected after the wave height calculations. These angles had standard deviations of less than $4^{\circ}$. The size of the standard deviations of $\phi, \theta$, and $\psi$ indicates that the error resulting from the small-angle form of the transformation matrix is small.

We have examined the effect of the transformation matrix of (3), that is, the tilt of the platform, on the wave height result. To investigate this, we have computed $z_{r}$, the height of the accelerometer in the rotating frame of the catamaran. This calculation is identical to that for $\eta_{\text {acc }}$, with the exception that the step given by (5) is omitted. This result is independent of the angles $\phi$ and $\theta$. We then compare the autospectra of the accelerometer heights in the nonrotating and rotating frames. In typical cases, the effect of the orientation angles increases with decreasing frequency and approaches about $10 \%$ of the spectral value at $f \simeq 0.1$ $\mathrm{Hz}$, suggesting that the rotational correction is indeed necessary.

\section{Estimation of directional frequency spectrum}

A wavenumber-frequency spectrum $\tilde{S}(\mathbf{k}, f)$ is calculated using the directional model discussed in this section. This model generalizes the original Data-Adaptive Spectral Estimator (DASE) model of Davis and Regier (1977) to allow the Doppler shifting caused by a moving platform. The reference frame used in the following sections has positive $x$ to the east, positive $y$ to the north, and positive $z$ upward.

\section{a. Doppler-shifted dispersion relation}

First, we describe the selection of the discrete wavenumbers $\mathbf{k}$ at which we compute the wavenumber-frequency spectrum. This is performed with separate re- quirements on the wavenumber magnitude $\kappa$ and the direction $\theta_{\kappa^{*}}$. The direction of $\mathbf{k}$ is discretized by using a constant spacing $\delta \theta=2 \pi / N_{\kappa \theta}$, where $N_{\kappa \theta}$ is an integer. The wavenumber magnitudes $\kappa$ are related to the observed frequency $f$ through the dispersion relation for small-amplitude waves with Doppler shifting,

$$
2 \pi f=\sqrt{g \kappa \tanh (\kappa H)}-U_{\mathrm{obs}} \kappa \cos \theta_{\mathrm{rel}} .
$$

Here, $g$ is the gravitational acceleration, $U_{\text {obs }}$ is the velocity of the observer relative to the mean current field, and $H$ is the water depth. The relative wave-observer angle $\theta_{\text {rel }}$ is defined according to

$$
\theta_{\text {rel }} \equiv \theta_{\kappa}-\theta_{\text {obs }}
$$

where $\theta_{\kappa}$ is the direction of $\mathbf{k}$ and $\theta_{\mathrm{obs}}$ is the heading of the observer relative to the mean current field. For convenience, we indicate the dispersion relation of (13) through the notation $\kappa=\kappa\left(f, \theta_{\kappa}\right)$.

The generalized DASE method requires solutions to the dispersion relation (13). We first consider the special case of deep water. In this case, $\tanh (\kappa H) \cong 1$ and solutions to (13) are given by

$$
\begin{aligned}
& \kappa=\frac{1}{2 U_{\mathrm{obs}}^{2} \cos ^{2} \theta_{\mathrm{rel}}} {\left[\left(g-4 \pi f U_{\mathrm{obs}} \cos \theta_{\mathrm{rel}}\right)\right.} \\
&\left. \pm \sqrt{g\left(g-8 \pi f U_{\mathrm{obs}} \cos \theta_{\mathrm{rel}}\right)}\right]
\end{aligned}
$$

for $\cos \theta_{\text {rel }} \neq 0$. The trivial solution for $\cos \theta_{\text {rel }}=0$ is

$$
\kappa=(2 \pi f)^{2} g^{-1} .
$$

Equations (14) and (15) present five solutions to the dispersion relation (13), as listed below in order of increasing $\kappa$ for fixed $f$.

$\kappa_{A} \quad(-)$ solution of (14) for $\cos \theta_{\text {rel }}<0$. Approaching waves.

$\kappa_{P}$ Solution of (15) for $\cos \theta_{\text {rel }}=0$. Waves moving perpendicularly.

$\kappa_{\mathrm{LT}}(-)$ solution of (14) $\cos \theta_{\text {rel }}>0$ and $f>0$. Long overtaking waves.

$\kappa_{\mathrm{ST}}(+)$ solution of (14) for $\cos \theta_{\text {rel }}>0$ and $f>0$. Short overtaking waves.

$\kappa_{R} \quad(+)$ solution of (14) for $\cos \theta_{\text {rel }}>0$ and $f<0$. Overrun waves.

The notations $(-)$ and $(+)$ refer to the choice of sign in front of the radical in (14). Solutions for overrun waves are found using negative frequencies $f$ in (14). These five solutions are shown in Fig. 6 for the example $U_{\text {rel }}=0.8 \mathrm{~m} \mathrm{~s}^{-1}$.

The three smallest-wavenumber solutions, $\kappa_{A}, \kappa_{P}$, and $\kappa_{\mathrm{LT}}$, are shown in Fig. 7 for $U_{\mathrm{obs}}=0.8 \mathrm{~m} \mathrm{~s}^{-1}$ and $\theta_{\mathrm{obs}}$ $=0^{\circ}$. Together, these solutions form closed curves in $\mathbf{k}$ space for small $f$. The two largest-wavenumber solutions, $\kappa_{\mathrm{ST}}$ and $\kappa_{R}$, correspond to waves with intrinsic frequencies much higher than the observed frequency $f$. These solutions may be ignored in the directional analysis if they correspond to waves with negligible energy compared to the other solutions. 


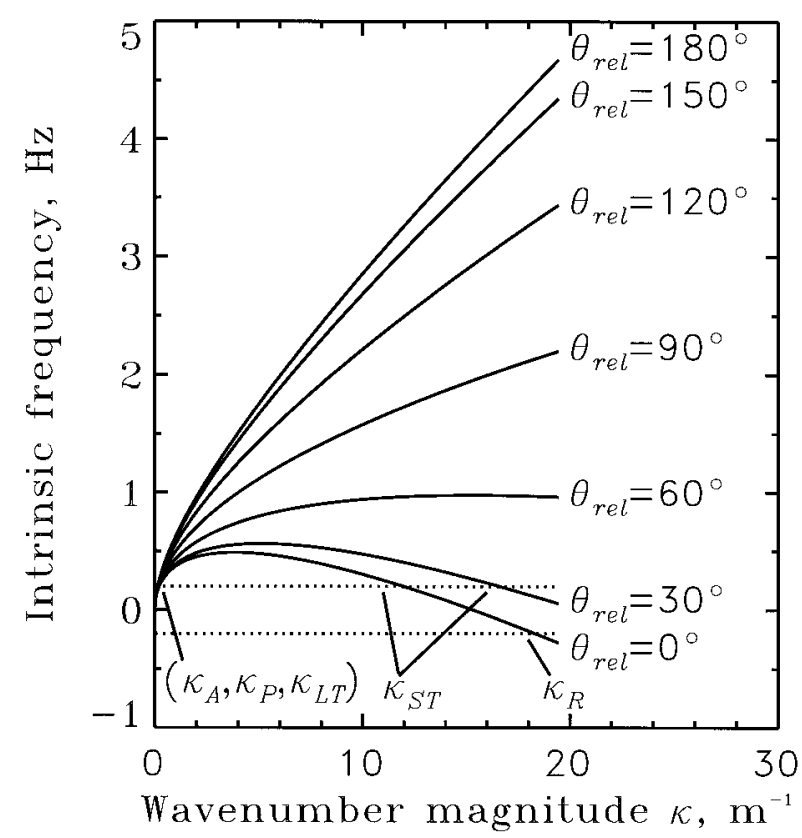

FIG. 6. Solutions to the Doppler-shifted dispersion relation (13) for deep water and $U_{\text {obs }}=0.8 \mathrm{~m} \mathrm{~s}^{-1}$. Dotted lines show the case of $f=$ $\pm 0.2 \mathrm{~Hz}$. The $\kappa$ solutions for $f= \pm 0.2 \mathrm{~Hz}$ are given by Eqs. (14) and (15).

The solution $\kappa_{\mathrm{LT}}$ is defined only for $f$ and $\theta_{\text {rel }}$, satisfying

$$
f \cos \theta_{\text {rel }} \leq f_{*}
$$

where

$$
f_{*}=\frac{g}{8 \pi U_{\mathrm{obs}}} .
$$

Thus, for frequencies $f$ above $f_{*}$, there is a range of $\theta_{\text {rel }}$ where no solution $\kappa_{\mathrm{LT}}$ exists. The curve corresponding to $f=0.58 \mathrm{~Hz}$ in Fig. 7 shows such a case. For $f>$ $f_{*}$, the ends of the curve given by $\kappa_{\mathrm{LT}}$ attach to the solution of $\kappa_{\mathrm{ST}}$. In practice, it is prudent to choose the observer velocity such that the waves of interest, for example, the dominant waves, have frequencies $f<f_{*}$.

When there is no Doppler shifting $U_{\mathrm{obs}}=0$, the dispersion relation (13) becomes

$$
g \kappa \tanh (\kappa H)=(2 \pi f)^{2} .
$$

Solutions for $\kappa$ from (17) describe circles in $\mathbf{k}$ space.

\section{b. Theoretical background}

Methods of estimating the propagation direction of waves generally utilize an approximation to the continuous integral

$$
S(\mathbf{k}, f)=\frac{1}{(2 \pi)^{2}} \int_{-\infty}^{\infty} Q(\boldsymbol{\zeta}, f) \exp (-i \mathbf{k} \cdot \boldsymbol{\zeta}) d \boldsymbol{\zeta},
$$

where $S(\mathbf{k}, f)$ is the wavenumber-frequency spectrum

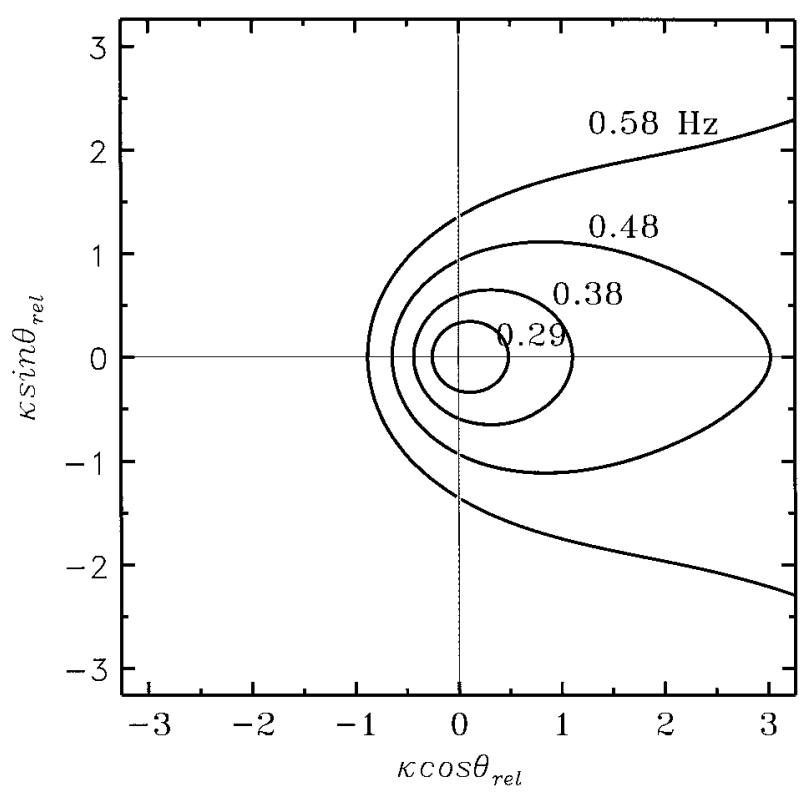

FIG. 7. The $\kappa_{\mathrm{A}}, \kappa_{P}$, and $\kappa_{\mathrm{LT}}$ solutions to the Doppler-shifted dispersion relation (13) for deep water and fixed $f$ of $0.29,0.38,0.48$, and $0.58 \mathrm{~Hz}$. Wavenumber magnitude $\kappa$ increases in the radial direction and propagation direction $\theta_{k}$ increases in the counterclockwise direction from the positive $x$ axis. Here, $U_{\mathrm{obs}}=0.8 \mathrm{~m} \mathrm{~s}^{-1}, \theta_{\mathrm{obs}}=0^{\circ}$. Together the three solutions form a closed loop only for $f<f_{*}=$ $0.49 \mathrm{~Hz}$.

and $\zeta$ is the lag vector between two locations in space. The term $Q(\zeta, f)$ is the cross spectrum between two height time series measured at locations separated by lag $\zeta$. The wavenumber $\mathbf{k}$ has magnitude $\kappa=2 \pi / a$ and direction $\theta_{\kappa}$, where $a$ is wavelength and $\theta_{\kappa}$ is the wave propagation direction. The calculation of the wavenumber-frequency spectrum applies to each observed frequency $f$ independently, and thus the dependence of all spectra on $f$ is implied throughout the following discussion. The angle $\theta_{\kappa}$ is measured in the horizontal plane, is zero along the $x$ axis, and increases in the counterclockwise direction when viewed from above.

The cross spectrum $Q(\boldsymbol{\zeta})$ is continuous in lag $\zeta$. But in practice, we estimate this quantity for discrete lags using the method in the appendix, yielding $\hat{Q}\left(\boldsymbol{\zeta}_{p q}\right)$. The discrete lag $\zeta_{p q}$ is the vector from wave wires $p$ to $q$, that is,

$$
\boldsymbol{\zeta}_{p q}=\mathbf{x}_{p}-\mathbf{x}_{q}
$$

The cross spectra are grouped in the matrix $\hat{\mathbf{Q}}$ with elements $\hat{Q}_{p q}=\hat{Q}\left(\boldsymbol{\zeta}_{p q}\right)$. Each of the diagonal elements $\hat{Q}_{\mathrm{pp}}$ for $p=1,2, \ldots, N_{\mathrm{ww}}$ is the autospectrum of wave height for the $p$ th wave wire and corresponds to the frequency spectrum $\hat{C}_{\eta \eta}$ discussed in section $3 \mathrm{~b}$. Here, $N_{\text {ww }}$ is the number of wave wires. The best estimate of the frequency spectrum $\hat{Q}_{a}$ is given by the average of the autospectra for the $N_{\text {ww }}$ wave wires;

$$
\hat{Q}_{a}=\frac{1}{N_{\mathrm{ww}}} \sum_{p=1}^{N_{\mathrm{ww}}} \hat{Q}_{\mathrm{pp}} .
$$


A simple approximation to (18) would be to replace $Q(\boldsymbol{\zeta})$ with the estimate $\hat{\mathbf{Q}}$ and to formulate an estimate of the differential $d \zeta$. However, given the sparse sampling in $\zeta$ space, the finite area elements corresponding to $d \zeta$ are poorly defined. We instead use a model in order to approximate (18). A general model can be written as

$$
\tilde{S}(\mathbf{k})=h \sum_{q=1}^{N_{\mathrm{ww}}} \sum_{p=1}^{N_{\mathrm{ww}}} \alpha_{\mathrm{pq}}(\mathbf{k}) \hat{Q}_{\mathrm{pq}},
$$

where $\alpha_{\mathrm{pq}}$ is an element of a matrix $\boldsymbol{\alpha}$ to be determined. The normalization factor $h$ ensures that the energy of $\hat{Q}_{a}$ is conserved in forming the estimate $\tilde{S}(\mathbf{k})$ and is discussed later. Next, we relate the model result $\tilde{S}(\mathbf{k})$ to the true spectrum $S(\mathbf{k})$ of (18). We first write the true cross spectrum $Q(\zeta)$ using the inverse of (18);

$$
Q(\boldsymbol{\zeta})=\int_{-\infty}^{\infty} S(\mathbf{l}) \exp (i \mathbf{l} \cdot \boldsymbol{\zeta}) d \mathbf{l},
$$

where we now use the wavenumber $\mathbf{l}$. To the accuracy of the estimate $\hat{Q}_{\mathrm{pq}}$, we may replace $\hat{Q}_{\mathrm{pq}}$ in (20) with its true value, $Q\left(\zeta_{\mathrm{pq}}\right)$, given in (21). This substitution yields

$$
\tilde{S}(\mathbf{k})=h \int_{-\infty}^{\infty} S(\mathbf{l}) w(\mathbf{k}, \mathbf{l}) d \mathbf{l},
$$

where

$$
w(\mathbf{k}, \mathbf{l})=\sum_{q=1}^{N_{\text {ww }}} \sum_{p=1}^{N_{\text {ww }}} \alpha_{\mathrm{pq}}(\mathbf{k}) \exp \left(i \mathbf{l} \cdot \boldsymbol{\zeta}_{\mathrm{pq}}\right) .
$$

The function $[h w(\mathbf{k}, \mathbf{l})]$ acts as the window function relating the estimated wavenumber-frequency spectrum to the true spectrum. This window must approximate a delta function centered on $\mathbf{l}=\mathbf{k}$.

\section{c. Fundamentals of the generalized DASE model}

The means of selecting the matrix $\boldsymbol{\alpha}$ varies from method to method. The DASE method of Davis and Regier (1977) was chosen for two reasons. First, it is a dataadaptive method. This implies that $\boldsymbol{\alpha}$ is selected with reference to the data $\hat{\mathbf{Q}}$ so that contributions to $\tilde{S}(\mathbf{k})$ from $\mathbf{I} \neq \mathbf{k}$ are minimized. This is in contrast to a priori methods, which attempt to minimize the sidelobe height of $w(\mathbf{k}, \mathbf{l})$ without regard to $\hat{\mathbf{Q}}$. Second, the DASE method constrains wave energy to lie on the dispersion curve $\kappa=\kappa\left(f, \boldsymbol{\theta}_{\kappa}\right)$. This dispersion relation is a strong physical restriction on waves. Other methods, such as the maximum likelihood method of Capon (1969), require no dispersion relation and thus allow undesired freedom in the calculation of $\tilde{S}(\mathbf{k})$.

The following three constraints are applied in both the original and generalized DASE methods.

1) The window $w(\mathbf{k}, \mathbf{l})$ is nonnegative.

2) Energy at a given frequency $f$ is distributed along the dispersion relation $\kappa=\kappa\left(f, \theta_{\kappa}\right)$.
3) The estimate $\tilde{S}(\mathbf{k})$ of least error is chosen from a restricted set of solutions.

Constraint 1 is imposed by requiring that the matrix $\boldsymbol{\alpha}$ be Hermitian, or equivalently,

$$
\boldsymbol{\alpha}(\mathbf{k})=\boldsymbol{\gamma}^{\mathrm{T}}(\mathbf{k}) \boldsymbol{\gamma}^{*}(\mathbf{k}),
$$

where the superscripts $\mathrm{T}$ and (asterisk) denote the matrix transpose and complex conjugate operations, respectively, and

$$
\boldsymbol{\gamma}=\left[\boldsymbol{\gamma}_{1}, \boldsymbol{\gamma}_{2}, \ldots, \boldsymbol{\gamma}_{N_{\mathrm{ww}}}\right] .
$$

The window $w(\mathbf{k}, \mathbf{l})$ of (23) may then be expressed as

$$
w(\mathbf{k}, \mathbf{l})=\boldsymbol{\gamma}(\mathbf{k}) \boldsymbol{\epsilon}^{* \mathrm{~T}}(\mathbf{l}) \boldsymbol{\epsilon}(\mathbf{l}) \boldsymbol{\gamma}^{* \mathrm{~T}}(\mathbf{k}),
$$

where $\boldsymbol{\epsilon}$ is the row vector

$$
\boldsymbol{\epsilon}=\left[e^{-i \mathbf{l} \cdot \mathbf{x}_{1}}, e^{-i \mathbf{l} \cdot \mathbf{x}_{2}}, \ldots, e^{-i \mathbf{l} \cdot \mathbf{x}_{N_{\mathrm{ww}}}}\right] .
$$

Equation (26) may be recast as

$$
w(\mathbf{k}, \mathbf{l})=\left|\boldsymbol{\gamma}(\mathbf{k}) \boldsymbol{\epsilon}^{* \mathrm{~T}}(\mathbf{l})\right|^{2} .
$$

This equation reveals that the window $w(\mathbf{k}, \mathbf{l})$ is nonnegative, that is, constraint 1 . This result is useful when combined with (22) in that the estimate $\tilde{S}(\mathbf{k})$ with least error corresponds to the minimum estimate.

\section{d. Generalized DASE window constraint}

The application of constraint 2 differs in the original and generalized DASE methods. In the generalized method, we require that the volume under the window $w(\mathbf{k}, \mathbf{l})$ integrated over the region $\Delta \mathbf{k}$ must remain constant with $\theta_{\kappa}$ for fixed $f$. The area $\Delta \mathbf{k}$ surrounds the point $\mathbf{k}$ in $\mathbf{k}$ space and is defined by the limits of integration in the analytical form of constraint 2 . This form is given by

$$
\int_{\theta_{k}-\Delta \theta / 2}^{\theta_{k}+\Delta \theta / 2} \int_{\lambda_{0}}^{\lambda_{1}} \lambda w(\mathbf{k}, \mathbf{l}) d \lambda d \theta_{l}=1,
$$

where $\gamma$ and $\theta_{l}$ are the magnitude and direction of $\mathbf{l}$, and $\Delta \theta$ is a constant angular width parameter specified later. The limits of the radial integral are defined using the dispersion relation (13), according to

$$
\begin{aligned}
& \lambda_{0} \equiv \kappa\left(f-\delta f / 2, \theta_{l}\right) \\
& \lambda_{1} \equiv \kappa\left(f+\delta f / 2, \theta_{l}\right),
\end{aligned}
$$

where $\delta f$ is the frequency resolution. Constraint 2 is needed because the area $\Delta \mathbf{k}$ varies with $\theta_{\kappa}$ for fixed $f$. This variation must be accounted for in the estimation of $\tilde{S}(\mathbf{k})$, which gives the spectral density per unit frequency and per unit area in $\mathbf{k}$ space.

For convenience, we recast (29) as

$$
\boldsymbol{\gamma}(\mathbf{k}) \mathrm{L}(\mathbf{k}) \boldsymbol{\gamma}^{* \mathrm{~T}}(\mathbf{k})=1,
$$

where $\mathbf{L}$ is a matrix with elements 


$$
L_{\mathrm{pq}}(\mathbf{k})=\int_{\theta_{k}-\Delta \theta / 2}^{\theta_{k}+\Delta \theta / 2} \int_{\lambda_{0}}^{\lambda_{1}} \exp \left(i \mathbf{I} \cdot \zeta_{\mathrm{pq}}\right) \lambda d \lambda d \theta_{l}
$$

In practice, we integrate (32) numerically using the trapezoidal rule, with 40 points across $\Delta \theta$ and 20 points in the radial direction $\gamma$ (see, e.g., Hornbeck 1975).

The choice for the angular width $\Delta \theta$ is arbitrary. We have tested the sensitivity of the results to its value. For the case of three wave wires, we have found negligible difference in peak height, peak location, and angular distribution of energy for values in the range $5^{\circ} \leq \Delta \theta$ $\leq 75^{\circ}$.

In the case without Doppler shifting, the radial integration limits of (32) are independent of $\theta_{\kappa}$, as described by (17). In this case, the constraint of (31) may be compared to the constraint in the original DASE method of Davis and Regier (1977). By neglecting the variation of $\mathbf{I} \cdot \boldsymbol{\zeta}_{\mathrm{pq}}$ in the radial direction $\lambda$, we may derive the following approximate form of (32):

$$
L_{\mathrm{pq}}(\mathbf{k})=\frac{1}{2}\left(\kappa_{1}^{2}-\kappa_{0}^{2}\right) \int_{\theta_{k}-\delta \theta / 2}^{\theta_{k}+\delta \theta / 2} \exp \left(i \mathbf{l} \cdot \zeta_{\mathrm{pq}}\right) d \theta_{l},
$$

where

$$
\begin{aligned}
& \kappa_{0} \equiv \kappa\left(f-\delta f / 2, \theta_{k}\right) \\
& \kappa_{1} \equiv \kappa\left(f+\delta f / 2, \theta_{k}\right) .
\end{aligned}
$$

The multiplication of the constant factor $\left(\kappa_{1}^{2}-\kappa_{0}^{2}\right) / 2$ by $\delta \theta$ gives the area of $\Delta \mathbf{k}$. By multiplying (33) by $2 \kappa /$ $\left(\kappa_{1}^{2}-\kappa_{0}^{2}\right)$, we obtain the constraint imposed in the original DASE method. Thus, the constraint of (31) modifies the original DASE constraint to account for the radial thickness in $\mathbf{k}$ space associated with the dispersion $\kappa=$ $\kappa\left(f, \theta_{\kappa}\right)$. This thickness is due to the discrete nature of the observed frequencies $f$ and is described by the boundaries given in (30).

\section{e. DASE maximization problem}

Next, we write the expression for $\tilde{S}(\mathbf{k})$ by combining (24) and (20), yielding

$$
\tilde{S}(\mathbf{k})=h \boldsymbol{\gamma}(\mathbf{k}) \hat{\mathbf{Q}} \boldsymbol{\gamma}^{* \mathrm{~T}}(\mathbf{k}) .
$$

The minimization of $\tilde{S}(\mathbf{k})$ is then equivalent to maximizing the ratio

$$
\nu(\mathbf{k})=\frac{h}{\tilde{S}(\mathbf{k})}=\frac{\boldsymbol{\gamma}(\mathbf{k}) \mathbf{L}(\mathbf{k}) \boldsymbol{\gamma}^{* \mathrm{~T}}(\mathbf{k})}{\boldsymbol{\gamma}(\mathbf{k}) \mathbf{Q} \boldsymbol{\gamma}^{* \mathrm{~T}}(\mathbf{k})} .
$$

The task is now to maximize $\nu$ under the condition

$$
\nu \boldsymbol{\gamma} \hat{\mathbf{Q}} \boldsymbol{\gamma}^{* \mathrm{~T}}=\boldsymbol{\gamma} \mathbf{L} \boldsymbol{\gamma}^{* \mathrm{~T}},
$$

where dependence on $\mathbf{k}$ is now implied. However, this maximization problem is not tractable. Instead, constraint 3 is imposed by maximizing $\nu$ subject to the condition

$$
\nu \hat{\mathbf{Q}} \boldsymbol{\gamma}^{* \mathrm{~T}}=\mathbf{L} \boldsymbol{\gamma}^{* \mathrm{~T}} .
$$

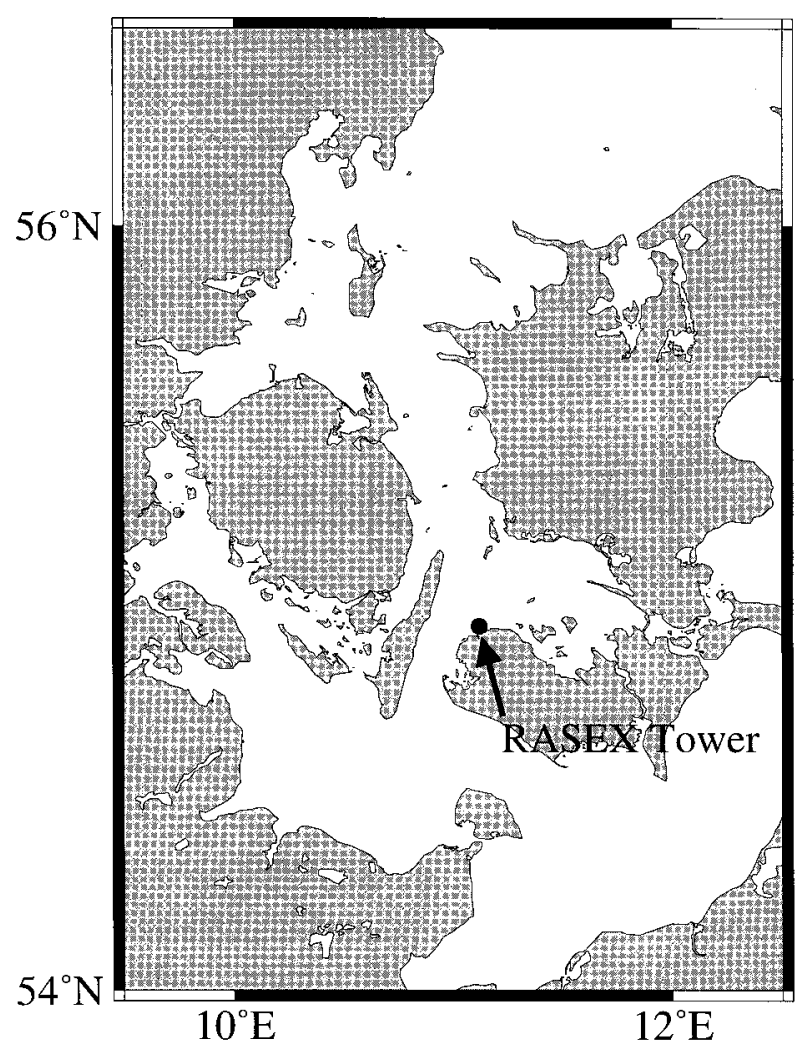

FIG. 8. The location of the RASEX experiment near the coast of Denmark. The research tower shown was outfitted with an array of three wave wires.

The cross-spectral matrix $\hat{\mathbf{Q}}$ is square and invertible. Multiplying (38) by $\hat{\mathbf{Q}}^{-1}$ yields

$$
\nu \boldsymbol{\gamma}^{* \mathrm{~T}}=\hat{\mathbf{Q}}^{-1} \mathbf{L} \boldsymbol{\gamma}^{* \mathrm{~T}} .
$$

This is a standard eigenvalue problem involving the given matrix $\hat{\mathbf{Q}}^{-1} \mathbf{L}$. The wavenumber-frequency spectrum is then

$$
\tilde{S}(\mathbf{k})=h / \nu_{\max }(\mathbf{k}),
$$

where $\nu_{\max }$ is the maximum eigenvalue.

The final requirement we place on $\tilde{S}(\mathbf{k})$ is that the energy over the area in $\mathbf{k}$ space corresponding to a particular $f$ must match the average autospectrum energy $\hat{Q}_{a}$; that is,

$$
\sum_{m=1}^{N_{k \theta}} \tilde{S}\left(\mathbf{k}_{m}\right) d A_{m}=\hat{Q}_{a},
$$

where $m$ is the index of the discrete $\mathbf{k}$ for fixed $f, \hat{Q}_{a}$ is given by (19), and $d A_{m}$ is the area given by

$$
d A=\int_{\theta_{k}-\delta \theta / 2}^{\theta_{k}+\delta \theta / 2} \int_{\lambda_{0}}^{\lambda_{1}} \lambda d \lambda d \theta_{l},
$$

for a particular $\mathbf{k}=\mathbf{k}_{m}$. Note that the area $d A$ differs from the area in the integral of (32) in that $\delta \theta$ is the 

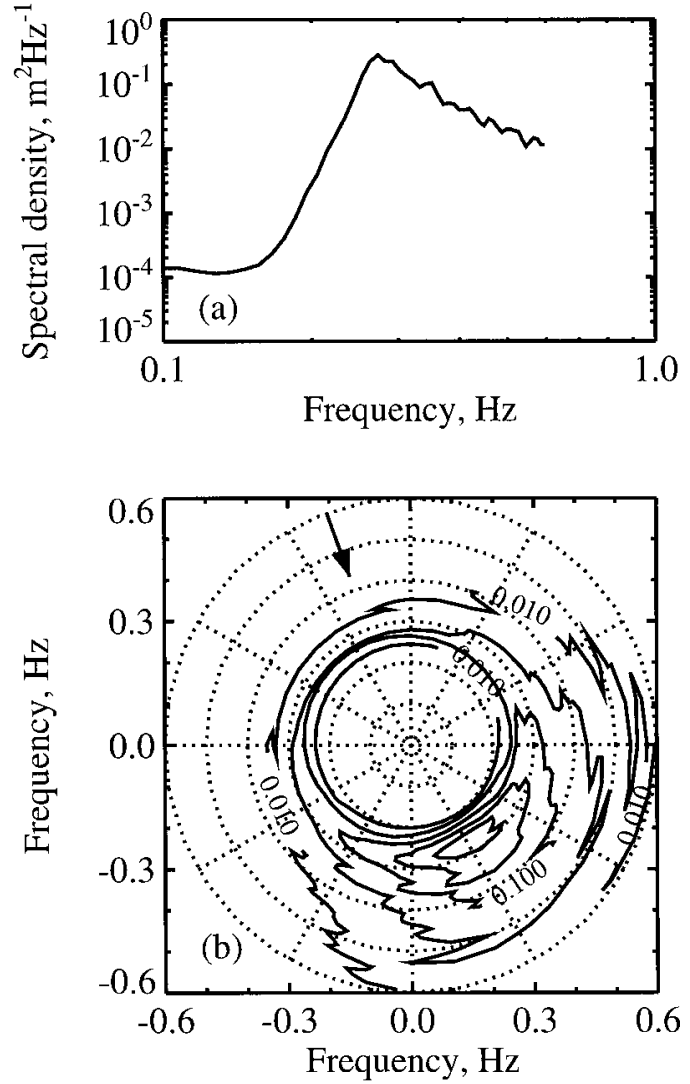

FIG. 9. RASEX wave-height spectral estimates for Julian day 121 from 0552 to 0622 UTC ( $P=34$ time blocks). (a) Frequency spectrum in meters squared per hertz. (b) Directional frequency spectrum, with intrinsic frequency (scale shown on axes) in the radial direction and wave propagation angle (north upward, east to the right) in the tangential direction. Contour labels are in units of meters squared per hertz per radians and adjacent contours have ratio $10^{1 / 2}$. Arrow represents wind direction; wind speed is $12 \mathrm{~m} \mathrm{~s}^{-1}$.

angular resolution while $\Delta \theta$ is a model parameter. Combining (40) with (41) yields

$$
h=\frac{\hat{Q}_{a}}{\sum_{m=1}^{N_{k \theta}} d A_{m} / \nu_{\max }\left(\mathbf{k}_{m}\right)} .
$$

The estimate $\tilde{S}(\mathbf{k})$ is then obtained by substituting (43) into (40).

\section{f. Calculation of the directional frequency spectrum}

The result $\tilde{S}$ obtained in the previous section is a threedimensional wavenumber-frequency spectrum. (Note that the $f$ dependence has been omitted for simplicity.) The spectrum $\tilde{S}(\mathbf{k}, f)$ is now converted to the two-dimensional wavenumber spectrum $\tilde{S}^{\prime}(\mathbf{k})$ according to

$$
\tilde{S}^{\prime}(\mathbf{k})=\delta f \sum_{j=1}^{N} \tilde{S}\left(\mathbf{k}, f_{j}\right)
$$

where $N$ is the number of discrete frequencies $f_{j}$. How-
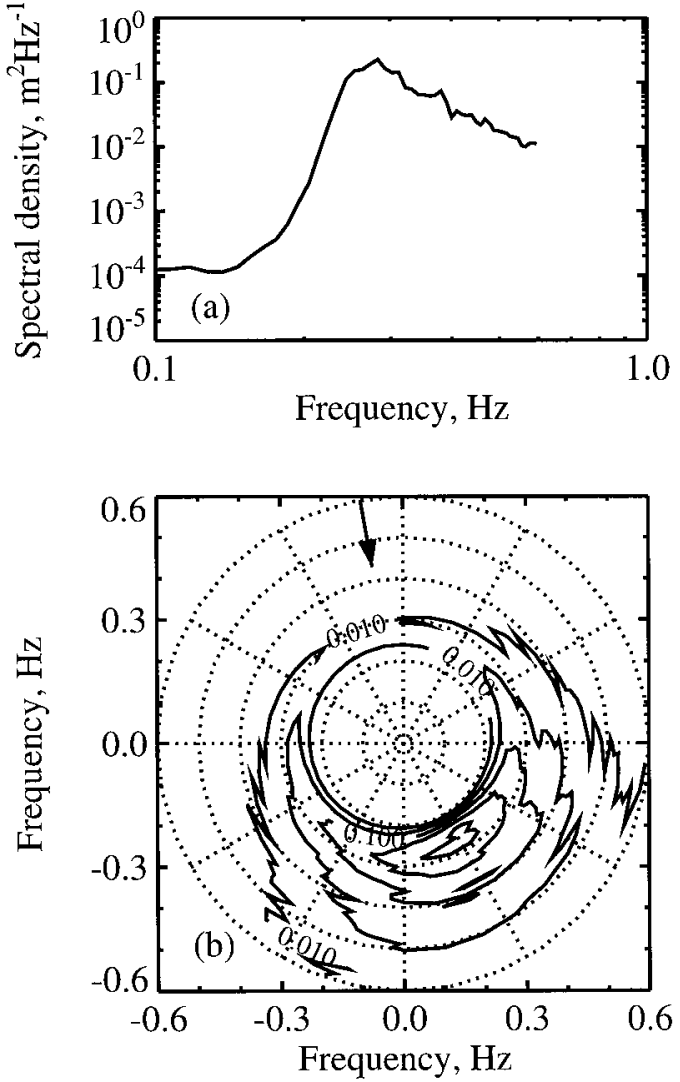

FIG. 10. RASEX wave-height spectral estimates, as in Fig. 9, but for Julian day 121 from 1035 to 1105 UTC ( $P=34$ time blocks). Wind speed is $8 \mathrm{~m} \mathrm{~s}^{-1}$.

ever, the DASE method distributes energy so that for a given $\mathbf{k}$ there is only one frequency bin $f_{k}$ that contributes nonzero energy. The relationship then simplifies to

$$
\tilde{S}^{\prime}(\mathbf{k})=\delta f \tilde{S}\left(\mathbf{k}, f_{k}\right) .
$$

Now we may compute the directional frequency spectrum $\tilde{D}\left(\tilde{f}, \theta_{k}\right)$. The wavenumber $\mathbf{k}$ is converted to intrinsic frequency $\tilde{f}$ using the following dispersion relation for small-amplitude waves:

$$
\tilde{f}=(2 \pi)^{-1} \sqrt{g \kappa \tanh (\kappa H)},
$$

which simplifies in the deep-water case to

$$
\tilde{f}=(2 \pi)^{-1}(g \kappa)^{1 / 2} .
$$

The directional frequency spectrum $\tilde{D}\left(\tilde{f}, \theta_{k}\right)$ is calculated via a conservation-of-energy constraint expressed by

$$
\kappa d \kappa \tilde{S}^{\prime}(\mathbf{k})=\tilde{D}\left(\tilde{f}, \theta_{k}\right) d \hat{f} .
$$

\section{Application of extended DASE method \\ a. Overview of RASEX experiment}

The Ris $\varnothing$ Air-Sea Experiment (RASEX) was sponsored by the Office of Naval Research as part of the 

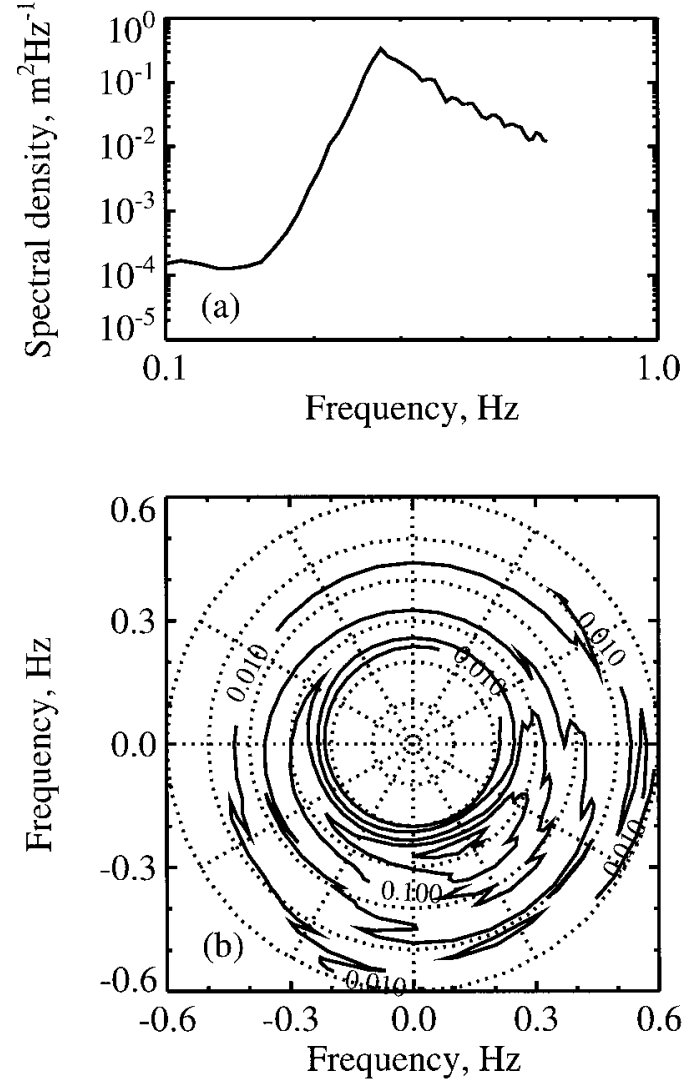

FIG. 11. Test of the angular resolution of the DASE method $(P=$ 34 time blocks). Spectral estimates of $\eta^{\prime \prime}$ in Eq. (48), with $c=0.33$. (a) and (b) as in Fig. 9.

Marine Boundary Layers Accelerated Research Initiative, and occurred in the Great Bælt off the western coast of Lolland, Denmark, in the spring and fall of 1994 (see Fig. 8). The data used for directional analysis were acquired from a $48-\mathrm{m}$ tower located in the nearshore waters. This tower was instrumented with an extensive array of meteorological sensors. The minimum and maximum fetches at this location were about 2 and $70 \mathrm{~km}$, and water depth was 3-4 m. The results discussed in section $5 \mathrm{~b}$ are from an array of three capacitive wave wires extended from the tower at the water surface. These wires formed the vertices of an equilateral triangle with sides $1 \mathrm{~m}$ in length.

\section{b. Directional frequency spectra results}

In this discussion, angles follow the meteorological convention, with zero northward and angle increasing in the clockwise direction. Wind directions are reported by the angle from which the wind blows, whereas wave angles describe the direction toward which waves propagate. Wave-height frequency spectra from the RASEX experiment were computed using 102-s time blocks and total time series lengths of $30 \mathrm{~min}$. Two directional spectra $\tilde{D}\left(\tilde{f}, \theta_{k}\right)$ for RASEX are shown in Figs. 9 and 10.
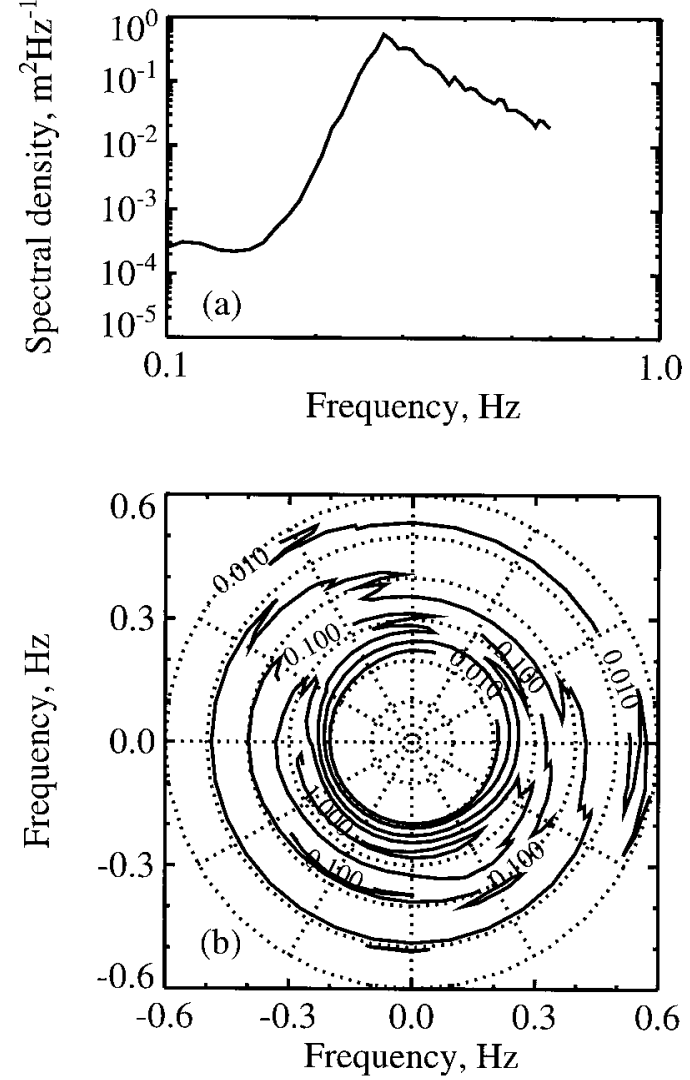

FIG. 12. Test of the angular resolution of the DASE method $(P=$ 34 time blocks). Spectral estimates of $\eta^{\prime \prime}$ in Eq. (48), with $c=1.0$. (a) and (b) as in Fig. 9.

In both figures, wind-wave peaks are shown to have angular locations closely aligned to the wind direction. The result of Fig. 9 corresponds to wind angle $\theta_{w}=$ $340^{\circ}$, wind speed $U_{w}=12 \mathrm{~m} \mathrm{~s}^{-1}$, and the wind-wave peak is located at $\tilde{f}=0.27 \mathrm{~Hz}$ and $\theta_{k}=145^{\circ}$. Its peak value is $\tilde{D}=2.4 \mathrm{~m}^{2} \mathrm{~Hz}^{-1} \mathrm{rad}^{-1}$. About $5 \mathrm{~h}$ later (Fig. 10), wind speed decreases to $U_{w}=8 \mathrm{~m} \mathrm{~s}^{-1}$, and the direction slightly shifts to $\theta_{w}=350^{\circ}$. Correspondingly, the spectral peak moves to $\tilde{f}=0.28 \mathrm{~Hz}$ and $\theta_{k}=160^{\circ}$, and its peak value is reduced to $\tilde{D}=1.6 \mathrm{~m}^{2} \mathrm{~Hz}^{-1} \mathrm{rad}^{-1}$. The difference between the wind and peak wave directions is $15^{\circ}$ and $10^{\circ}$ for Figs. 9 and 10 , respectively. Since the wind and peak-wave directions may differ slightly in the presence of variable fetch (Donelan et al. 1985), as in RASEX, the observed correlations seem reasonable.

The accuracy of the directional method is undoubtedly a function of the ratio of wavelength to wave-wire spacing. The peaks found at 0.28 and $0.45 \mathrm{~Hz}$ correspond to wavelengths $a=2 \pi / \kappa$ of 17 and $8 \mathrm{~m}$, respectively. The spacing between any two wires in the RASEX array is $1 \mathrm{~m}$. Thus, the ratios of peak wavelength to wave-wire spacing are 17 and 8 , and the directional method apparently performs well throughout this range. 

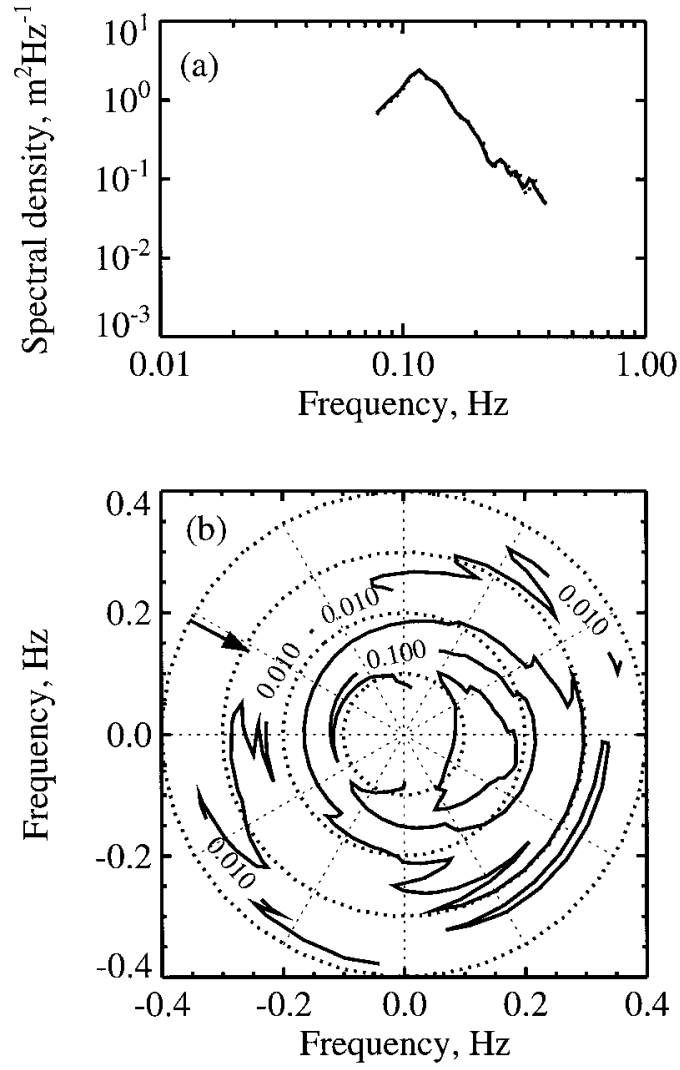

\section{c. Numerical experiment}

A test is conducted to investigate the performance of the directional method in resolving a secondary wave peak with a different direction than that of dominant waves. We first synthesize wave-wire time series that contain two different dominant wave systems, by combining two sets of experimental data taken $5 \mathrm{~h}$ apart. Let $\left(\eta_{1}, \eta_{2}, \eta_{3}\right)$ be the three wave-wire signals used in the calculation of the result of Fig. 9 , and let $\left(\eta_{1}^{\prime}, \eta_{2}^{\prime}\right.$, $\left.\eta_{3}^{\prime}\right)$ be the three wave-wire signals used for Fig. 10 . Note that these results have peak directions about $15^{\circ}$ apart. We form a new set of wave-wire time series by combining these two sets according to the relation

$$
\eta_{m}^{\prime \prime}=\eta_{m}+c \frac{\eta_{\mathrm{rms}}}{\eta_{\mathrm{rms}}^{\prime}} \eta_{n}^{\prime}
$$

where $c$ is a constant in the range $0 \leq c \leq 1$, and the indices $m=1,2$, and 3 correspond to the indices $n=$ 3,1 , and 2 , respectively. We define the index of $\eta$ so that it increases in the clockwise direction. Thus, we have shifted the wave-wire data of the $\eta^{\prime}$ set by $120^{\circ}$ in the clockwise direction. The root-mean-square heights $\eta_{\mathrm{rms}}$ and $\eta_{\mathrm{rms}}^{\prime}$ are defined according to

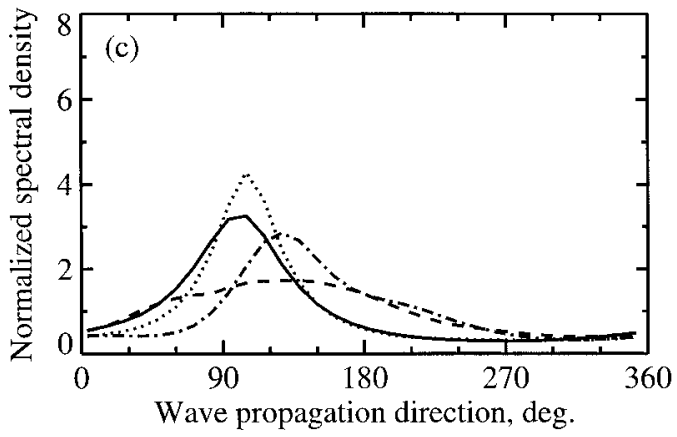

FIG. 13. CoOP wave-height spectral estimates for Julian day 132 from 1546 to 1625 UTC ( $P=43$ time blocks). (a) Frequency spectrum in meters squared per hertz. Solid line: intrinsic frequency; dotted line: observed frequency. (b) Directional frequency spectrum, with intrinsic frequency (scale shown on axes) in the radial direction and wave propagation angle (north upward, east to the right) in the tangential direction. Contour labels are in units of meters squared per hertz per radians and adjacent contours have ratio $10^{1 / 2}$. Arrow represents wind direction; wind speed is $4.5 \mathrm{~m} \mathrm{~s}^{-1}$. (c) Normalized directional spreading at different frequencies. Solid line: $0.1 \mathrm{~Hz}$; dotted line: $0.2 \mathrm{~Hz}$; dashed line: $0.3 \mathrm{~Hz}$; dash-dot line: $0.4 \mathrm{~Hz}$.

$$
\begin{aligned}
& \eta_{\mathrm{rms}}=\left[\frac{1}{3 N} \sum_{m=1}^{3} \sum_{j=1}^{N} \eta_{m}^{2}\left(t_{j}\right)\right]^{1 / 2} \\
& \eta_{\mathrm{rms}}^{\prime}=\left[\frac{1}{3 N} \sum_{n=1}^{3} \sum_{j=1}^{N} \eta_{n}^{\prime 2}\left(t_{j}\right)\right]^{1 / 2},
\end{aligned}
$$

where $N$ is the number of discrete times $t_{i}$ used in the directional estimate. The ratio $\eta_{\mathrm{rms}} / \eta_{\mathrm{rms}}^{\prime}$ in (48) scales the energy in the time series $\eta_{n}^{\prime}$ to match that of $\eta_{m}$.

The directional frequency spectra of the $\eta^{\prime \prime}$ time series are computed for values of the constant $c$ of 0.33 and 1.0, with the results shown in Figs. 11 and 12. Additionally, Fig. 9 may be interpreted as the $c=0$ case. As $c$ increases, the angular domains of the 0.3 and 0.1 $\mathrm{m}^{2} \mathrm{~Hz}^{-1} \mathrm{rad}^{-1}$ contours increase in the clockwise direction. Asymmetry is evident even at the $c=0.33$ level. Figure 12 should correspond to the sum of Fig. 9 and Fig. 10 (after rescaling) rotated by $120^{\circ}$ if the time series $\eta$ and $\eta^{\prime}$ are uncorrelated, which is true in this case. The true peaks due to $\eta$ and $\eta^{\prime}$ should be located at about $145^{\circ}$ and $280^{\circ}$, respectively, while Fig. 12 shows two peaks at about $160^{\circ}$ and $255^{\circ}$. Despite the slightly decreased angular separation between the two peaks in the latter case, the presence of two peaks in the $c=1$ case shows that the generalized DASE method is capable of resolving a bimodal spectrum with 

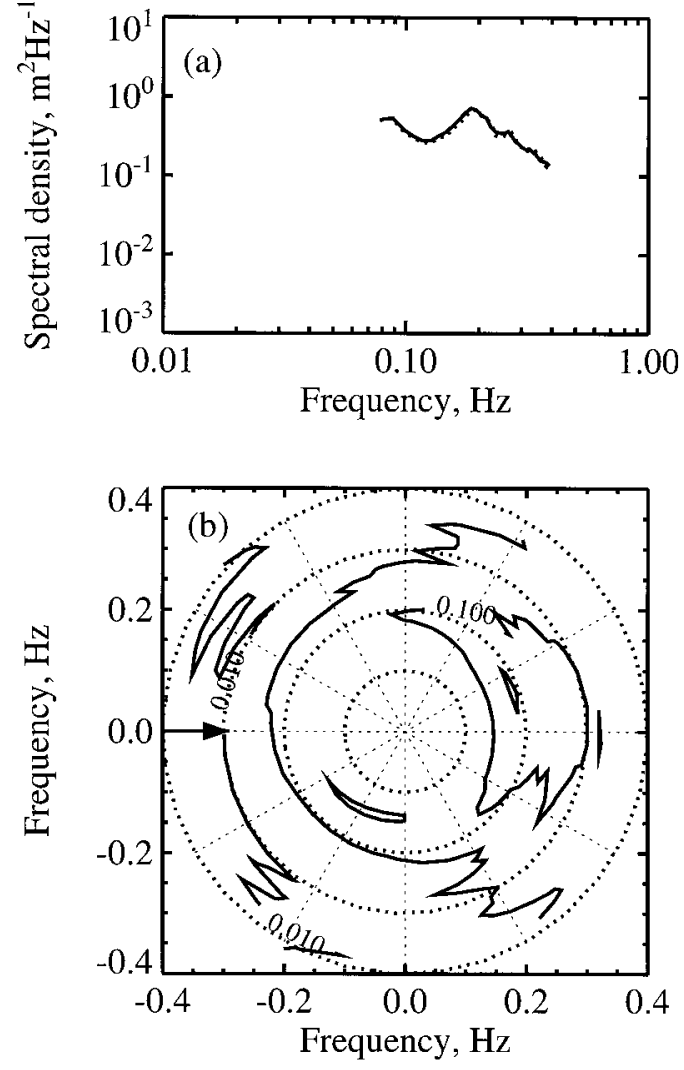

similar peak energy and peak angles separated by $125^{\circ}$. Furthermore, the asymmetry of contours in the $c=0.33$ cases indicates that the method is sensitive enough to respond to the presence of significantly smaller secondary waves separated in angle from dominant waves. This allows the generalized DASE method to be used in detecting the energy of reflected waves from the RASEX tower posts (Hare 1995).

\section{Application of motion correction scheme and extended DASE method}

In this section we combine the motion correction scheme discussed in section 2 and the extended DASE method developed in section 4. Using signals obtained from a towed research catamaran during the CoOP experiment, directional wave spectra are estimated.

\section{a. Overview of $\mathrm{CoOP}$ experiment}

The Coastal Ocean Processes (CoOP) experiment occurred in April-May 1995, off the California coast, and was sponsored by the National Science Foundation. The principal objective of the program was to investigate the role of various ocean surface processes on the airsea gas exchange. The research catamaran was towed to the side of the R/V New Horizon. Measurements of gravity-capillary waves, surface gravity waves, sub-

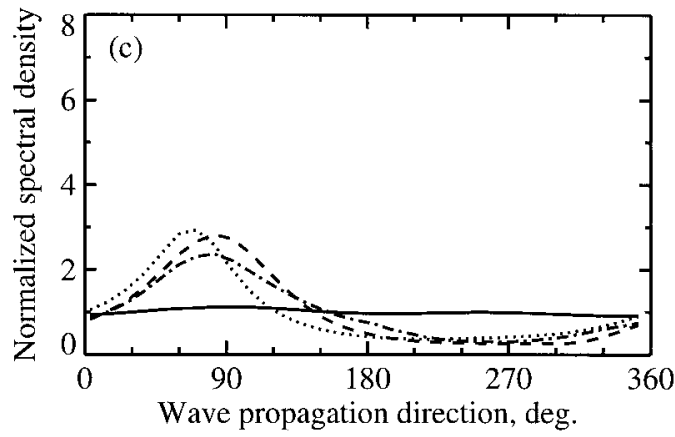

FIG. 14. CoOP wave-height spectral estimates for Julian day 133 from 1844 to 1930 UTC ( $P=52$ time blocks). (a) Frequency spectrum in meters squared per hertz. Solid line: intrinsic frequency; dotted line: observed frequency. (b) Directional frequency spectrum, with intrinsic frequency (scale shown on axes) in the radial direction and wave propagation angle (north upward, east to the right) in the tangential direction. Contour labels are in units of meters squared per hertz per radians and adjacent contours have ratio $10^{1 / 2}$. Arrow represents wind direction; wind speed is $9 \mathrm{~m} \mathrm{~s}^{-1}$. (c) Normalized directional spreading at different frequencies. Solid line: $0.1 \mathrm{~Hz}$; dotted line: $0.2 \mathrm{~Hz}$; dashed line: $0.3 \mathrm{~Hz}$; dash-dot line: $0.4 \mathrm{~Hz}$.

surface currents-turbulence, and surface enrichment were made possible with the instrument array aboard the catamaran. This array included a set of six capacitive wave wires, a motion detection package, a three-axis acoustic Doppler current meter, a hot film anemometer, and a surface skimmer. The motion detection package consisted of a three-axis translational accelerometer and a three-axis angular-rate sensor. Both the wave-wire data and the motion detection package data were recorded digitally at a sampling rate of $100 \mathrm{~Hz}$. The wind speed, direction, and stress were obtained at the bow of the ship using a sonic anemometer.

\section{b. Directional frequency spectra results}

The procedure for estimating the wave-height spectrum for CoOP was identical to the procedure used during High-Res 2. Because no independent measurements of the wave-height spectrum were available, we cannot validate the spectra for CoOP as we did for High-Res 2. In Figs. 13-15, the results of frequency spectra, directional frequency spectra, and normalized directional spreading are shown for three cases. Wave-height frequency spectra for Figs. 13-15 were computed using 102-s time blocks and total time series of 39, 46, and 45 mins, respectively. All measurements were performed in the vicinity of Santa Catalina Island, shown in Fig. 16. The first two figures correspond to cases 

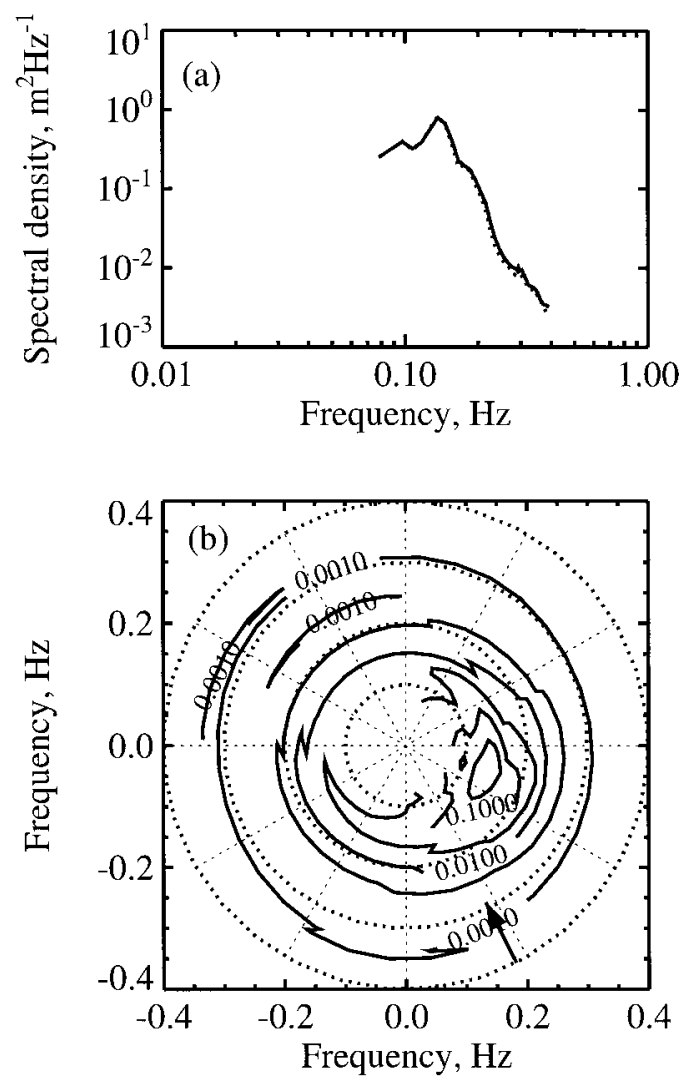

where wind and swells are aligned. Figure 13 shows an example of well-developed swells and relatively low local wind waves under weak wind. The directional spreading of swells $(0.1-0.2 \mathrm{~Hz})$ is narrower than wind waves $(0.3-0.4 \mathrm{~Hz})$ as expected. On the other hand, the wave field in Fig. 14 is mostly local wind waves generated by strong wind. The low level of swells may be partially due to the sheltering effect of the island. In

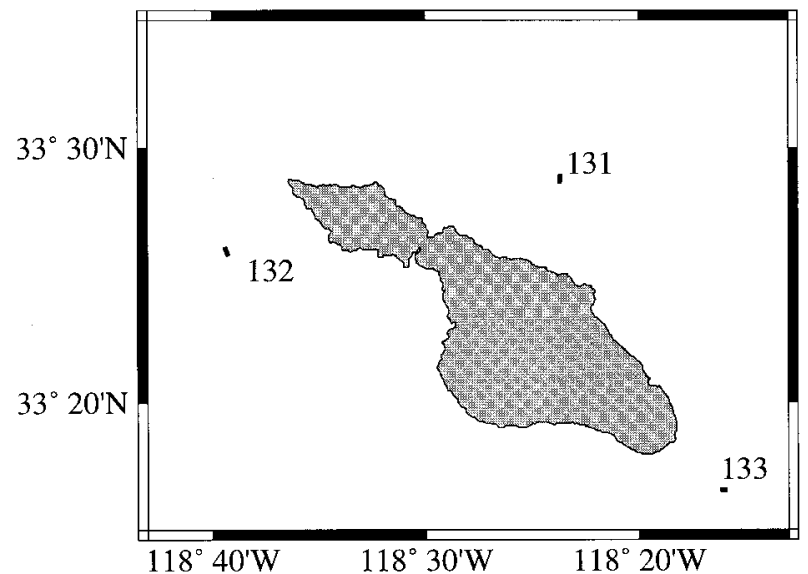

FIG. 16. The location of the $\mathrm{CoOP}$ wave measurements of this study was near Santa Catalina Island.

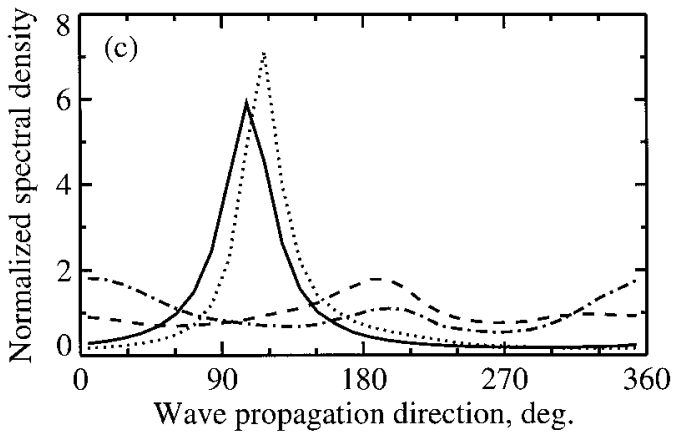

FIG. 15. CoOP wave-height spectral estimates for Julian day 131 from 0409 to 0454 UTC ( $P=51$ time blocks). (a) Frequency spectrum in meters squared per hertz. Solid line: intrinsic frequency; dotted line: observed frequency. (b) Directional frequency spectrum, with intrinsic frequency (scale shown on axes) in the radial direction and wave propagation angle (north upward, east to the right) in the tangential direction. Contour labels are in units of meters squared per hertz per radian and adjacent contours have ratio $10^{1 / 2}$. Arrow represents wind direction; wind speed is $4 \mathrm{~m} \mathrm{~s}^{-1}$. (c) Normalized directional spreading at different frequencies. Solid line: $0.1 \mathrm{~Hz}$; dotted line: $0.2 \mathrm{~Hz}$; dashed line: $0.3 \mathrm{~Hz}$; dash-dot line: $0.4 \mathrm{~Hz}$.

both figures, the directions of local wind waves are consistent with the observed wind directions.

An example is shown in Fig. 15 where swells and local wind waves are not aligned. In this case, the wind speed is relatively low and both wind speed and wind direction are rather variable. At swell frequencies $(0.1-$ $0.2 \mathrm{~Hz})$ the directional spreading is narrow and well defined. At the highest frequency $(0.4 \mathrm{~Hz})$, the peak direction is roughly consistent with the local wind but the wave energy is more widely spread in all directions. In between at $0.3 \mathrm{~Hz}$, the wave field appears to be confused because of the presence of both swells and local wind waves.

These examples demonstrate that the combination of our motion correction scheme and the extended DASE method may successfully estimate directional wave spectra, including both swells and local wind waves in the range of wave conditions encountered during $\mathrm{CoOP}$. A more comprehensive analysis of the accuracy of this method in a wider range of conditions is a topic for future research.

\section{Conclusions}

We have developed a method to obtain directional spectra of surface gravity waves using a motion detection package and a wave-wire array, both mounted on 
a moving platform. The validity of the motion correction scheme to the wave-wire signals has been confirmed by comparison with independent buoy observations. An improved method of computing the directional frequency spectrum has been derived by generalizing the DASE method of Davis and Regier (1977) to allow for the motion of the observer relative to the wave field. The applications of the method to two field datasets, one from a fixed platform and the other from a moving platform, have shown that the propagation direction of the dominant wind waves agree well with the wind direction. Furthermore, the swell direction at lower frequencies can be resolved accurately even if it is not aligned with local wind. A numerical test with the fixed platform data demonstrates sufficient sensitivity of the model to detect the presence of secondary waves propagating at different incidence angles than those of dominant waves.

The directional wave information obtained in this study can be directly correlated with other collocated physical measurements of atmospheric turbulence, short wind waves, and subsurface turbulence in order to address detailed interaction between various air-sea interaction processes and surface gravity waves. Currently, we are using the directional spectra of wind waves in a study of their modulation of shorter gravitycapillary waves.

Acknowledgments. We thank Drs. James Edson and Wade McGillis for providing meteorological information. Dr. Edson also provided valuable information regarding the motion correction scheme. Dr. Hans Graber generously provided wave-height spectra from buoy measurements. This research was supported by ONR Grant N00014-93-1-1214 (KAH). Partial support was provided by ONR Grant N00014-95-1-0116 (TH) and N00014-90-J-1717 (EJB).

\section{APPENDIX}

\section{Estimation of Frequency Spectra}

The Welch method is a standard means of computing cross spectra and utilizes overlapping time blocks and nonrectangular data windows (Marple 1987). The Welch estimate of the cross spectrum is given by

$$
\hat{C}_{12}\left(f_{k}\right)=\frac{1}{\mu T P} \sum_{p=1}^{P} X_{1}\left(f_{k}, p\right) X_{2}^{*}\left(f_{k}, p\right),
$$

where the asterisk denotes the complex conjugate operation, the summation is over $P$ time blocks, each of duration $T$, and $X_{1}$ and $X_{2}$ are the Fourier transforms of the time signals $x_{1}(t)$ and $x_{2}(t)$. If $x_{2}(t)=x_{1}(t)$, then the result $\hat{C}_{11}\left(f_{k}\right)$ is the autospectrum of $x_{1}(t)$. The constant $\mu$ is described shortly. The discrete, finite Fourier transforms (DFTs) $X_{1}\left(f_{k}, p\right)$ and $X_{2}\left(f_{k}, p\right)$ are given by the general relation

$$
X\left(f_{k}\right)=\frac{1}{f_{\text {samp }}} \sum_{n=0}^{N-1} x\left(t_{n}\right) w\left(t_{n}\right) e^{-i 2 \pi k n / N},
$$

where $N$ is the number of discrete points in the time block, $f_{\text {samp }}$ is the sampling frequency in hertz, and $x\left(t_{n}\right)$ is the discrete time series. The discrete frequencies $f_{k}$ are given by

$$
f_{k}=f_{\text {samp }} k / N \quad \text { with } \quad k=0,1, \ldots, N-1 .
$$

In practice, the DFTs are computed using the fast Fourier algorithm (see, e.g., Bendat and Piersol 1986).

We have generally chosen $w\left(t_{n}\right)$ to be the Hann window (Marple 1987);

$$
w_{h}\left(t_{n}\right)=\cos ^{2}\left[\pi \frac{n-(N-1) / 2}{N-1}\right] .
$$

This window has smaller sidelobes than those of the simple rectangular window $w_{r}\left(t_{n}\right)=1$. Sidelobes are a phenomenon of discrete Fourier analysis causing the power at one frequency to be spread to neighboring frequencies. Although the Hann window has smaller sidelobes than the rectangular window, it has a slightly wider mainlobe peak. This mainlobe width determines the effective resolution of the spectrum. The rectangular window has an effective mainlobe width equal to the frequency spacing of (A3), or $\delta f=f_{1}=f_{\text {samp }} / N$. For the Hann window, this resolution is $1.5 \delta f$.

The constant $\mu$ in (A1) compensates for the loss in total energy of the time signal $x\left(t_{n}\right)$ due to the windowing operation. It is defined for all windows by

$$
\mu=\frac{1}{N} \sum_{n=0}^{N-1} w\left(t_{n}\right)
$$

For the Hann window, $\mu=0.37$.

The use of overlapping time blocks in the calculation of $\hat{C}_{12}\left(f_{k}\right)$ increases $P$ and therefore the statistical stability of the estimate. It is justified by the form of the Hann window. Consider two successive time blocks of length $N$. Signals near their common edge are damped heavily by Hann window values close to zero, as shown by (A4), with $n$ approaching 0 or $N-1$. Thus, their contribution to the DFT of (A2) is small. In an overlapping block centered on this edge, these oscillations are windowed with $w_{h}\left(t_{n}\right)$ close to 1 and will make a significant contribution to the DFT. The sum of the window values $w_{h}\left(t_{n}\right)$ of one block and the values of the two overlapping windows is, in fact, identically equal to 1 . Thus, only the first and last $N / 2$ points of the entire time series are underutilized.

\section{REFERENCES}

Bendat, J. S., and A. G. Piersol, 1986: Random Data. 2d ed. WileyInterscience, $566 \mathrm{pp}$.

Bock, E. J., and N. M. Frew, 1993: Static and dynamic response of natural multicomponent oceanic surface films to compression and dilation: Laboratory and field observations. J. Geophys. Res., 98, $14599-14617$. 
- and Coauthors, 1995: Description of the scientific plan for the April 1995 CoOP experiment, 'Gas transfer in coastal waters,' performed from the research vessel New Horizon. Air-Water Gas Transfer, B. Jaehne and E. C. Monahan, Eds., AEON Verlag and Studio, 801-810.

Capon, J., 1969: High-resolution frequency-wavenumber spectrum analysis. Proc. IEEE, 57, 1408-1418.

Carlson, D. J., J. L. Cantey, and J. J. Cullen, 1988: Description and results from a new surface microlayer sampling device. DeepSea Res., 35, 1205-1213.

Davis, R. E., and L. A. Regier, 1977: Methods for estimating directional wave spectra from multi-element arrays. J. Mar. Res., 35, 453-477.

Donelan, M. A., J. Hamilton, and W. H. Hui, 1985: Directional spectra of wind-generated waves. Philos. Trans. Roy. Soc. London, Ser. A, 315, 509-562.

Drennan, W. M., M. A. Donelan, N. Madsen, K. B. Katsaros, E. A. Terray, and C. N. Flagg, 1994: Directional wave spectra from a swath ship at sea. J. Atmos. Oceanic Technol., 11, 1109-1116.

Hanson, K. A., 1996: Open-ocean observation of the modulation of gravity-capillary waves by long gravity waves. M.S. thesis,
Graduate School of Oceanography, University of Rhode Island, 80 pp. [Available from Graduate School of Oceanography, University of Rhode Island, Narragansett, RI 02882.]

Hara, T., E. J. Bock, and D. Lyzenga, 1994: In situ measurements of capillary-gravity wave spectra using a scanning laser slope gauge and microwave radars. J. Geophys. Res., 99, 12 593-12 602.

Hare, J., 1995: A similarity analysis of the structure of the waveinduced flow fields. Ph.D. thesis, The Pennsylvania State University, $165 \mathrm{pp}$.

Hornbeck, R. W., 1975: Numerical Methods. Quantum, 310 pp.

Kats, A. V., and I. S. Spevak, 1980: Reconstruction of the sea-wave spectra from measurements of moving sensors. Izv. Acad. Sci. USSR, Atmos. Oceanic Phys., 16, 194-200.

Longuet-Higgins, M. S., D. E. Cartwright, and N. D. Smith, 1963: Observations of the directional spectrum of sea waves using the motion of a floating buoy. Ocean Wave Spectra, Prentice-Hall, 111-132.

Macomber, G. R., and M. Fernandez, 1962: Inertial Guidance Engineering. Prentice-Hall, $530 \mathrm{pp}$

Marple, S. L., 1987: Digital Spectral Analysis with Applications. Prentice-Hall, 492 pp. 